\title{
Effects on post-glacial rebound from the hard rheology in the transition zone
}

\author{
Giorgio Spada, ${ }^{1}$ Roberto Sabadini, ${ }^{1,2}$ David A. Yuen ${ }^{3}$ and Yanick Ricard ${ }^{4}$ \\ ${ }^{1}$ Dipartimento di Fisica-Settore Geofisica, Università di Bologna, Viale B. Pichat 8, 40127 Bologna, Italy \\ ${ }^{2}$ Istituto di Mineralogia, Università di Ferrara, 44100 Ferrara, Italy \\ ${ }^{3}$ Minnesota Supercomputer Institute and Department of Geology and Geophysics, University of Minnesota, Minneapolis, MN 55415, USA \\ ${ }^{4}$ Département de Geologie, Ecole Normale Supérieure, 75231 Paris, France
}

Accepted 1992 January 6. Received 1991 December 30; in original form 1991 March 28

\begin{abstract}
SUMMARY
We analyse the influences of a viscosity increase in the transition zone between 420 and $670 \mathrm{~km}$ on the geophysical signatures induced by post-glacial rebound, ranging from the perturbations in the Earth's rotation to the short wavelength features associated with the migration of the peripheral bulge. A seif-gravitating model is adopted, consisting of an elastic lithosphere, a three-layer viscoelastic mantle and an inviscid core.

The horizontal displacements and velocities and the stress pattern are extremely sensitive to the viscosity increase and to the chemical stratification of the transition zone. The hardening of the upper and the chemical density jumps in mantle below the 420 discontinuity induces a channel effect which contaminates the horizontal deformation both in the near-field and in the far-field from the ice-sheets. These findings indicate that intraplate geodetic data can be used to put bounds on the viscosity increase in the transition zone and on the amount of chemical stratification in the mantle.

The stress field induced in the lithosphere by the Pleistocenic ice-sheet disintegration is a very sensitive function of mantle viscosity stratification. The existence of seismic activity along passive continental margins of previously glaciated areas requires a substantial viscosity increase in the mantle, with the viscosity of the transition zone acting as a controlling parameter. A viscously stratified mantle is responsible for a delayed upward migration of stress in the lithosphere which can account for the seismicity today.
\end{abstract}

Key words: post-glacial rebound, transition zone, viscosity.

\section{INTRODUCTION}

The problem of mantle viscosity stratification has important consequences in many areas of geodynamics and geochemistry, as it touches on issues related to the style of mantle convection and geochemical mixing. There have been many experimental studies on the rheological properties of the upper mantle (e.g. Mackwell, Kohlstedt \& Paterson 1985; Karato, Paterson \& Fitzgerald 1986; Mackwell, Bai \& Kohlstedt 1990). In contrast, our understanding of deformation in the deep mantle is rather incomplete because there are few experimental constraints on the rheology of the transition zone and lower mantle. Recent laboratory studies of key mantle minerals have suggested that the transition zone may form a layer of relatively high viscosity oetween the upper and lower mantle (Karato 1989; Meade \& Jeanloz 1990). Recently Ricard, Vigny \& Froidevaux (1989) in a Monte-Carlo non-linear inversion analysis have found that models with a stiff layer in the transition zone produce acceptable surface plate velocities. A hard layer also serves to decouple the geoid responses of the lower mantle from those due to the upper mantle density heterogeneities (Colin \& Fleitout 1990). Evidence for the existence of a hard transition zone in the mantle also comes from seismology (e.g. Anderson \& Bass 1986; Montagner \& Anderson 1989; Dost 1990; Gubbins \& Snieder 1991). Such a layer is intrinsically harder than olivine and perovskite (Karato 1989; Meade \& Jeanloz 1990). The presence of a hard layer at the base of the upper mantle would have tremendous influences on the ability to retrieve mantle 


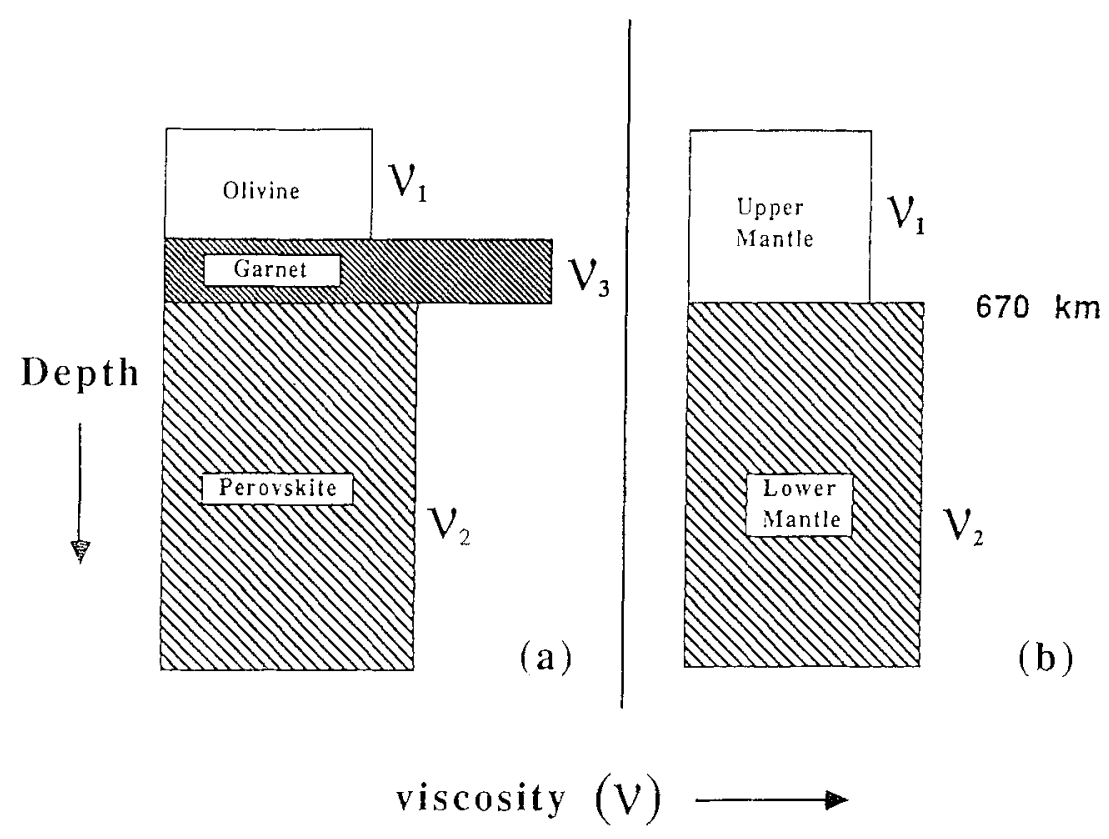

Figure 1. Schematic diagram depicting the two rheological models. (a) The three-layer mantle model with the hard garnet layer present in the transition zone between 420 and $670 \mathrm{~km}$ depth; the viscosity in this middle layer is denoted by $v_{3}$ while $v_{1}$ and $v_{2}$ represent respectively the upper and lower mantle viscosities. (b) The traditional two-layer model with the $670 \mathrm{~km}$ discontinuity dividing the upper and lower mantles.

viscosity structures from post-glacial rebound and geoid anomalies. Such a hard layer can also greatly influence convective flows through the many phase changes in the transition zone (Anderson 1987). It will certainly affect the stress distributions within the subducting slab and consequently the clustering of earthquakes in the deep portions of descending slabs. Non-equilibrium effects from sluggish kinetics of the phase transitions (Morris 1992) in slabs can also induce a vorticity field that opposes the flow and makes the transition zone globally stiffer than for equilibrium phase boundaries. In fact, slow kinetic effects from phase transitions in slabs would make the phasetransition boundaries behave like chemical boundaries. Recent inversion of seismic $Q$ data also indicates an increase of $Q$ at $400 \mathrm{~km}$ depth, indicating a change to a stiffer rheology for short time-scales (Widmer, Masters \& Gilbert 1991). Therefore, it is important to investigate the dynamical influences from a high-viscosity transition zone on geodynamical signatures. Viscous modelling of this hard layer has already demonstrated that different dynamical behaviour can be expected from the contrast of rheologies across the transition zone (Hong, Yuen \& Wu 1990; Wu \& Yuen 1991). Preliminary results from a spherical viscoelastic model (Spada, Sabadini \& Yuen 1991a) have also corroborated the importance of including the hard layer. In this work we investigate far more extensively the various dynamical effects arising from this hard layer in the transition zone within the framework of forward modelling. The body of this paper is organized as follows. We first describe the rheological model with the hard layer present. This is followed by a mathematical description of the five-layer viscoelastic model where the forcing function comes from Pleistocene deglaciation. We then give the results for the vertical and horizontal displacements, gravity anomaly fields, stress fields, and rotational signatures.
Finally we discuss the overall geodynamical implications of this hard layer in the transition zone.

\section{A RHEOLOGICAL MODEL FOR THE TRANSITION ZONE}

In geodynamical modelling one commonly assumes that the viscosity profile below the asthenosphere increases up the $D^{\prime \prime}$ layer, where there may be a low-viscosity zone (e.g. Yuen \& Peltier 1980). The rheology of the mantle, on the other hand, depends on crystal structure, as well as on temperature and pressure. Some authors (Anderson \& Bass 1986; Anderson 1989; Duffy \& Anderson 1989) have suggested that the transition zone of the mantle, between 420 and $670 \mathrm{~km}$ depth, is different, in terms of mineralogy and chemistry, from both the upper and lower mantle. On the basis of the systematic relationship between hightemperature plasticity and crystal structure, Karato (1989) has argued for the geophysical significance of the garnet layer in its rheological comparison to the olivine and perovskite layers in the mantle. In Fig. 1(a), we present the viscosity profile for the three-layer mantle; $v_{1}$ is the olivine (upper mantle) viscosity, $v_{2}$ refers to the perovskite (lower mantle) and $v_{3}$ to the garnet layer. In Fig. 1(b), the traditional two-layer model is shown, $v_{1}$ and $v_{2}$ being respectively the upper and lower mantle viscosities (Wu \& Peltier 1982; Yuen, Sabadini \& Boschi 1982). The parameter $C=v_{3} / v_{1}$ expresses the hardness of the garnet with respect to the olivine layer. The elastic moduli and densities are also different in each of the layers and are taken from seismic estimates. From systematics of dense oxides Karato (1989) has shown that the ratio $C$ should be between $O(10)$ and $O(100)$; we will vary this ratio in examining certain values of $v_{2} / v_{1}$. 


\section{MATHEMATICAL DESCRIPTION OF THE FIVE-LAYER MODEL}

The possible presence of a rheological discontinuity in the transition zone of the mantle, from 420 to $670 \mathrm{~km}$ depth, requires an improvement over previous Earth models, based on a two-layer viscoelastic mantle (Sabadini, Yuen \& Boschi 1982; Yuen et al. 1982). We present here the analytical mathematical description of a self-gravitating, incompressible Earth model consisting of an elastic lithosphere, a three-layer Maxwell viscoelastic mantle and an inviscid core. The main result we have obtained here is the explicit expression for the Green's function of the problem, i.e. for the solution associated to with point mass located at the surface of the Earth and tidal loading. The correspondence principle for linear viscoelasticity (e.g. Fung 1965) allows us to deal with elastic equations, where the elastic shear modulus is substituted by $\mu(s)=\mu s /(s+\mu / v)$ in order to take into account the viscoelastic mantle rheology. According to this principle, the time dependence of the problem is recovered by evaluating the inverse Laplace transform of the $s$-dependent solution. Each of the viscoelastic layers is characterized by four parameters: the shear modulus $\mu$, the dynamic viscosity $v$, the density $\rho$ and the thickness. The core consists of an inviscid fluid with density $\rho_{C}$ and the elastic lithosphere is described by a shear modulus $\mu_{L}$ and a density $\rho_{L}$. Realistic values for these parameters are given in Table 1.

For an Earth model whose mechanical properties are only functions of radius $r$, the linearized equation of momentum conservation, the constraint of incompressibility and the Poisson equation for the perturbed geopotential can be developed in series of orthogonal Legendre polynomials $P_{l}(\cos \theta)$ and put in the form of a set of six linear first-order ordinary differential equations (Longman 1962; Farrell 1972; Peltier 1974; Sabadini, Yuen \& Boschi 1984a). The solution vector for a degree $l, \mathbf{y}^{\prime}(r, s)$ of the above set of differential equations contains as unknowns the radial and lateral components of the displacement scalar field, the normal and shear stresses, the perturbed gravitational potential and an auxiliary variable $q^{\prime}$ related to the gravity field ('potential traction')

$\mathbf{y}^{l}(r, s)=\left(U^{l}, V^{l}, \sigma_{r r}^{l}, \sigma_{r \theta}^{l},-\phi^{l}, q^{l}\right)^{\mathbf{T}}$

(Sabadini et al. 1982; Yuen et al. 1982). The solution vector within each viscoelastic mantle layer and within the elastic lithosphere can be expressed by using a fundamental matrix $Y^{\prime}(r, s)$ (Wu 1978; Sabadini et al. 1982)

$\mathbf{y}^{\prime}(r, s)=Y^{\prime}(r, s) \mathbf{C}^{\prime}(s)$.

The unknowns $\mathbf{C}^{\prime}(s)$ can be determined by writing the continuity equations of the six field variables at each layer

Table 1. Physical properties of the Earth employed in modelling.

Parameter Value $(\mathrm{km})$ Parameter Value $\left(\mathrm{kg} / \mathrm{m}^{3}\right)$ Parameter Value $\left(\mathrm{N} / \mathrm{m}^{2}\right)$

$\begin{array}{cccccc}a & 6371 & \rho_{L} & 4120 & \mu_{L} & 7.28 \times 10^{10} \\ b & 6271 & \rho_{1} & 4120 & \mu_{1} & 9.54 \times 10^{10} \\ r_{2} & 5951 & \rho_{3} & 4220 & \mu_{3} & 1.1 \times 10^{11} \\ r_{1} & 5701 & \rho_{2} & 4508 & \mu_{2} & 1.99 \times 10^{11} \\ c & 3480 & \rho_{C} & 10925 & \mu_{C} & 0\end{array}$

interface and by fitting the appropriate set of boundary conditions at the surface of the Earth and at the core-mantle boundary. If we denote the radii of the Earth, of the lithosphere-upper mantle boundary, of the interface at 420 and $670 \mathrm{~km}$ depth and of the core-mantle boundary by $a, b, r_{2}, r_{1}$ and $c$ (Table 1), the whole set of boundary equations, for each angular order $l$, can be reduced to the following form:

$O(s) \mathbf{C}_{C}(s)=\mathbf{B}_{f}$,

where the forcing vector $\mathbf{B}_{f}$ depends on whether one is considering tidal loading (Takeuchi, Saito \& Kobayashi 1962 ) or surface point mass loading (Farrell 1972). The $3 \times 3$ matrix $O(s)$ is given by

$$
\begin{aligned}
O(s)= & M_{L}(a) Y_{L}^{-1}(b) Y_{1}(b) Y_{1}^{-1}\left(r_{2}\right) Y_{3}\left(r_{2}\right) \\
& \times Y_{3}^{-1}\left(r_{1}\right) Y_{2}\left(r_{1}\right) Y_{2}^{-1}(c) I_{c}(c) .
\end{aligned}
$$

We dropped, for the sake of simplicity, the $s$ dependence on the right side and the subscripts $L, 1,3,2$ and $C$ indicate that the correspondent quantities pertain respectively to the lithosphere, the upper mantle, the transition zone, the lower mantle and the core. The $6 \times 3$ interface matrix $I_{c}(c)$ (Yuen et al. 1982), takes into account the fluid-solid boundary conditions at the core-mantle boundary (Chinnery 1975; Crossley \& Gubbins 1975), while the $3 \times 6$ matrix $M_{L}(a)$ is built in such a way as to satisfy the external surface boundary conditions (Sabadini et al. 1982). Effects due to a fluid core rotating relative to a viscoelastic mantle have recently been considered by Lefftz \& Legros (1992).

By means of the algebraic manipulator Mathematica (Wolfram et al. 1988; Spada et al. 1990) we have inverted analytically the fundamental matrix $Y(r, s)$ (see the Appendix) in order to obtain an explicit form of $O(s)$. An explicit form of the $O(s)$ matrix can be found by partitioning both the direct and inverse matrices into an 'elastic' and a 'viscous' part

$$
\begin{aligned}
& Y_{\mathrm{dir}}(r, s)=Y_{\mathrm{dir}}^{E}(r)+\left(s+\mu_{k} / v_{k}\right)^{-1} Y_{\mathrm{dir}}^{V}(r), \\
& Y_{\mathrm{inv}}(r, s)=Y_{\mathrm{inv}}^{E}(r)+s^{-1} Y_{\mathrm{inv}}^{V}(r),
\end{aligned}
$$

where $\mu_{k} / v_{k}$ is the inverse Maxwell relaxation time of the $k$ th viscoelastic layer. The elements of the matrices defined by (5a) and (5b) are derived from the fundamental matrix $Y(r, s)$ given in the Appendix (see the Appendix for additional details). We use (5a) and (5b) in (4) and (3) to arrive at the following result:

$\mathbf{C}_{C}(s)=s^{3}\left[\prod_{j=1}^{3}\left(s+\mu_{j} / v_{j}\right)\right]\left(\sum_{k=0}^{6} s^{k} O_{k}\right)^{-1} \mathbf{B}_{f}$.

The seven $3 \times 3$ numerical matrices $O_{k}$ are derived by the expansion of (4). We denote the matrix enclosed in square brackets in (6) by $P(s)$. Since each of its elements is a degree six polynomial in $s$, its determinant can be found easily using the properties of the Ricci Levi-Civita alternating tensor $\epsilon_{i j k}$ :

$|P(s)|=\sum_{\alpha, \beta, \gamma=0}^{6} s^{\alpha+\beta+\gamma} \sum_{i, j, k=1}^{3} \epsilon_{i j k} p_{1 i}^{(\alpha)} p_{2 j}^{(\beta)} p_{3 k}^{(\gamma)}$,

where $p_{i j}^{(k)}$ is the coefficient of the $k$ th power of $s$ in the $(i, j)$ element of $P(s)$.

Finally, we give the formal expression of the canonical 


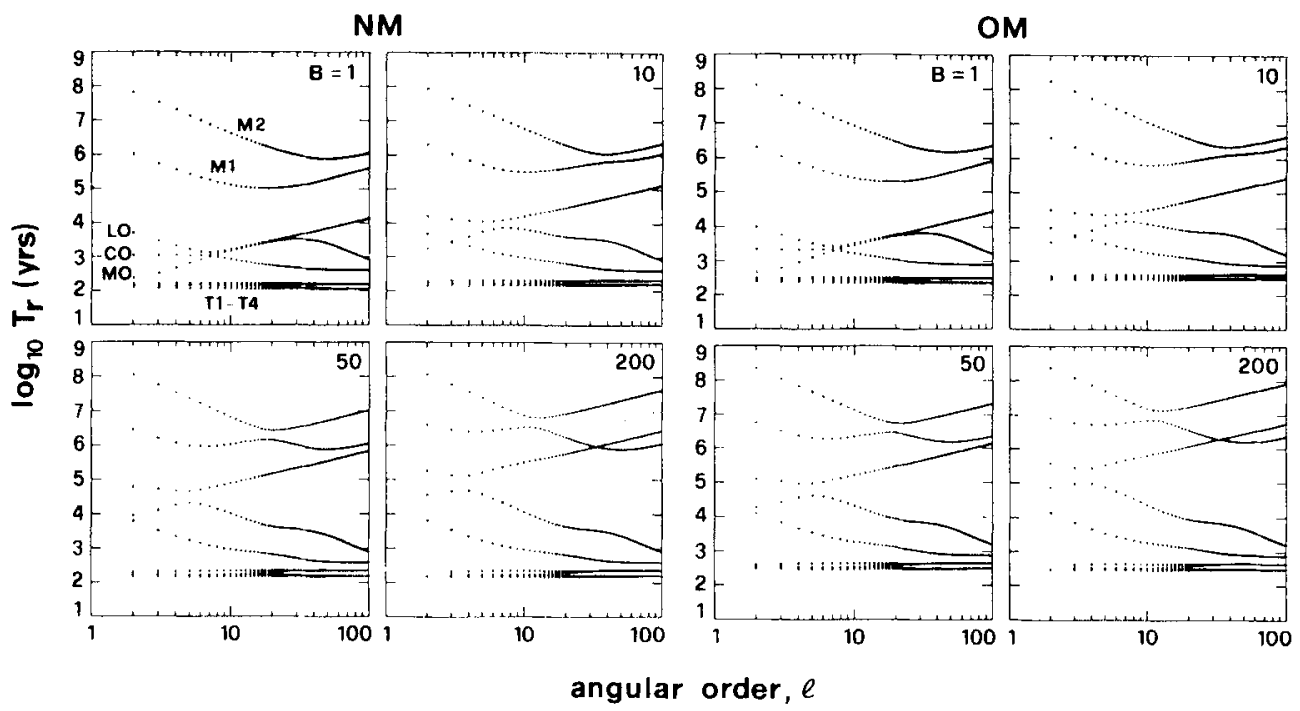

Figure 2. Relaxation time as a function of the harmonic order $l$ and varying lower mantle viscosity. $B$ is varied from 1 to 200 , for the old model OM with $v_{1}=10^{21}$ Pa s (e.g. Wu \& Peltier 1982) and the new model NM with $v_{1}=5 \times 10^{20}$ Pas (e.g. Ricard \& Sabadini 1990).

dispersion relation for the five-layer model, obtained by equating equation (7) to zero:

$\sum_{\alpha, \beta, \gamma=0}^{6} s^{\alpha+\beta+\gamma} D_{\alpha \beta \gamma}=0$.

The 343 coefficients $D_{\alpha \beta \gamma}$ are determinants involving $3 \times 3$ constant matrices and are computed directly by a subroutine. Although formally the secular equation (8) involves a 18th degree polynomial (see Spada et al. 1990), calculations have shown that the factors of the five lower powers of the variable $s$ are negligible. We can thus classify the roots in the following way: six null roots, three roots equal to the Maxwell times $\mu_{j} / v_{j}$ of each viscoelastic layer and nine real and negative roots $s_{i}$ (inverse relaxation times). It is important to note that, by definition, the quantities $s_{i}$ do not depend on the type of forcing that perturbs the planet (tidal or point mass loading): they are intrinsic functions of the physical parameters of the model and of the harmonic degree. The viscoelastic relaxation spectra for various Earth viscosity profiles are discussed as follows. From backsubstitution of (6) in (2) through (4) we can derive a spectral decomposition for the different Green's functions $G(r, \theta, s)$ in the Laplace domain. These Green's functions can be, for example, the gravity field or the velocities induced by a unit excitation function. Their general form is the same as for the three- and four-layer models, the only difference being the number of relaxation modes

$G(r, \theta, s)=\sum_{l}\left(G^{E}(r)+\sum_{i=1}^{9} \frac{G_{i}^{V}(r)}{s-s_{i}}\right) P_{i}(\cos \theta)$

where $E$ and $V$ denote respectively elastic and viscous contributions. Standard methods in Laplace inversion and space-time convolution are used to evaluate the responses of the planet to time-dependent loads of finite size (Farrell 1972; Wu \& Peltier 1982). To ensure numerical convergence, up to 100 terms $(l=100)$ have been used. The most commonly studied signatures of glacial isostasy are the surface deformations and their associated free-air gravity anomalies. Two additional signatures are useful to constrain the internal mantle viscosity structure. These are the present-day polar wander (Lambeck 1980; Dickman 1981) and the current secular variation of the degree two zonal coefficient of the gravitational potential $\dot{J}_{2}$ (Yoder et al. 1983; Cheng et al. 1989). We can employ the isostatic and rotational eigenspectra of the five-layer model for calculating these rotational signatures of our viscoelastic planet (Sabadini, Yuen \& Boschi 1984b). Analytical formulae for the secular variations of the other gravity potential coefficients (zonal; $J_{l}$ and azimuthal; $C_{l m}$ and $S_{l m}$ ), from both point-source and finite-sized discs have been developed by Sabadini, Yuen \& Gasperini (1988). They can also be applied within the framework of this new five-layer model.

\section{VISCOSITY INCREASE IN THE LOWER MANTLE AND IN THE HARD LAYER: EFFECTS ON THE RELAXATION TIMES AND MODAL DEFORMATION}

The effects of radial viscosity variations in the mantle for the relaxation times of the different viscoelastic modes are shown in Figs 2 and 3. The relaxation times $T_{r}$ are expressed in years as a function of the harmonic degrees $l$ ranging from 2 to 100 . In Fig. 2 we first deal with a viscosity increase in the lower mantle. The ratio $B=v_{2} / v_{1}$ between the lower and upper mantle viscosities is varied from 1 to 200 . OM stands for the old viscosity models with $v_{1}=10^{21} \mathrm{~Pa}$ s (Yuen et al. 1982; Wu \& Peltier 1982) while NM corresponds to the new models with $v_{1}=0.5 \times 10^{21} \mathrm{Pas}$, as in Table 2, consistent with the new inferences of upper mantle viscosity from geoid anomalies (Ricard et al. 1989) and post-glacial rebound analyses (Lambeck, Johnston \& Nakada 1990). The viscosity in the whole upper mantle is uniform. The mantle is chemically stratified at 420 and $670 \mathrm{~km}$ depth and thus supports nine relaxation modes.

The two slowest modes, called M2 and M1 (Wu \& Peltier 1982) are associated with the two internal chemical boundaries. At low degrees, they are followed by the 


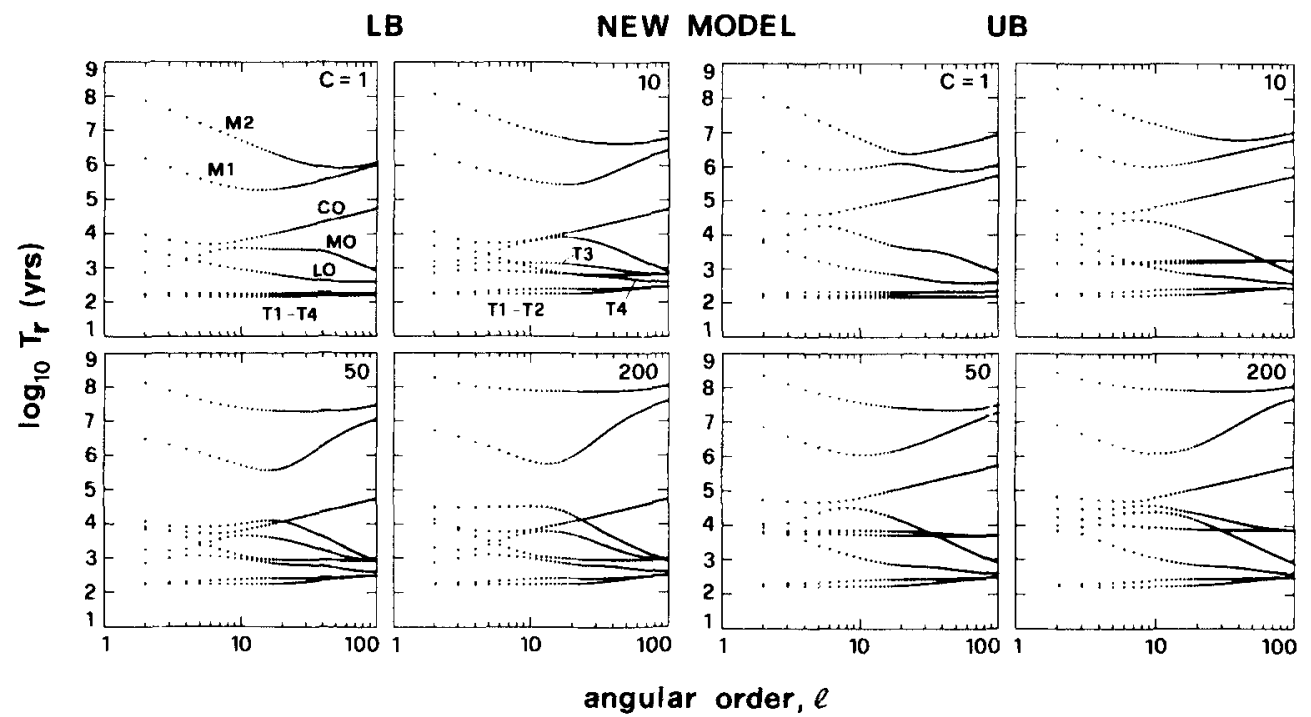

Figure 3. As in Fig. 2, except the viscosity in the transition zone for the new model NM is varied accordingly to $C=1,10,50,200$. The left and right columns, denoted by LB and UB stand for the lower and upper branch solutions.

Table 2. Mantle viscosity profiles employed in modelling.

Model

Lower Branch $(L B)$

Upper Branch (UB)

OLD (OM)

$$
\nu_{1}=10^{21} \text { Pas } \quad \nu_{1}=10^{21} \text { Pas }
$$

$$
\nu_{2}=10^{21} \quad \nu_{2}=4 \times 10^{22}
$$

$$
\nu_{1}=0.5 \times 10^{21} \quad \nu_{1}=0.5 \times 10^{21}
$$

NEW (NM)

$$
\nu_{2}=2 \times 10^{21} \quad \nu_{2}=2 \times 10^{22}
$$

lithospheric (LO), the core (CO) and mantle (MO) modes, as portrayed in the panel for $B=1, \mathrm{NM}$. The fastest modes at the bottom correspond to the transient T-modes (Spada et al. 1990). The three Maxwell times are not included in this figure. The effects of parameter variations on the relaxation times can be obtained from comparison with the labelled curves of panel $B=1$, NM.

When we increase the lower mantle viscosity from $B=1$ to $B=200$ all the curves are moved upward to slower relaxation times. The upward migration of the curves occur first for the longer wavelengths $l<10$, followed by the shorter ones which are less affected by the lower mantle viscosity. For $l>20$ only the M1, M2 and core modes have slower relaxation times. For these high angular degrees the deformation concentrated near the surface. Therefore the lithospheric and mantle modes are basically unaffected by a viscosity increase in the lower mantle.

We note that the curves for the new model NM in the left panel can be deduced from their counterparts in the right panel by a uniform downward shift toward faster relaxation times, in agreement with the lower values of the mean mantie viscosity. The reduction of the averaged mantle viscosity by a factor of 2 reduces the relaxation times by the same amount.
Fig. 3 portrays the effects of a viscosity increase in the hard layer for the new reference models NM given in Table 2 that will be used throughout. The family NM is, in turn, subdivided into a lower branch LB characterized by a smooth viscosity increase in the lower mantle, and an upper branch UB with $v_{2}=40 v_{1}$. This terminology is based on the multiple lower mantle viscosities which can reproduce rebound observations (Sabadini \& Peltier 1981; Peltier 1983; Yuen et al. 1986). The ratio $C=v_{3} / v_{1}$ is varied from 1 to 200 to display the full sensitivity of the relaxation times to viscosity variations in the hard layer. The modes can be identified by the labelling of panels $C=1, C=10$, LB.

$A$ viscosity increase in the hard layer drastically influences the whole family of modes for all the models. In particular the $M 1$ and $M 2$ modes are more influenced than the other modes. The most striking difference from the findings of the previous figure stands on the fact that both long and short wavelengths are simultaneously affected by variations in the parameter $C$. The hard layer lies close to the surface where the deformation of the shorter wavelengths is concentrated. Also the OM model would appear to be influenced in a similar fashion by a viscosity increase in the hard layer.

A better physical insight can be obtained from the analysis of the radial and tangential deformation induced by a pure spherical harmonic loading function of degree $l=2$ and $l=9$, as portrayed in Figs $4($ a) and (b) respectively for the new model NM of Table 2 . For each of the nine relaxation modes, characteristic of a chemically stratified mantle at 420 and $670 \mathrm{~km}$, the left and right portions of the rings depict the radial and tangential components of the displacement field in the lithosphere and in the mantle. Red and blue colours denote positive and negative quantities. The radial scale accounts for the relative correct thicknesses between the different layers. We note that the amplitude of each mode is normalized to one, so that the relative strength between the different modes cannot be compared. The characteristic relaxation times are given in $10^{3}$ years $(\mathrm{kyr})$ increasing from the left to the right and from the top to the bottom. The effects of the garnet layer are analysed in the bottom panels of Figs 4(a) and (b). In each panel the first 
four modes are associated with fast transient relaxation modes that do not carry a substantial fraction of the deformation energy for loading boundary conditions (Yuen et al. 1986). The last two modes are characteristic of the M1 and $\mathrm{M} 2$ modes.

For both the harmonics $l=2$ and $l=9$, a viscosity increase at $670 \mathrm{~km}$ in the UB models has the tendency to inhibit the deformation in the lower mantle, both radial and tangential. The viscosity increase acts as a shield, focusing the deformation in the outer viscoelastic layer. This effect is particularly important for the $l=2$ harmonic, that penetrates deeper in the lower mantle for the LB models. For the higher degree harmonic $l=9$, this effect is smoother because this component samples in any case the outer part of the mantle. This finding indicates that the secular variations of the higher degree harmonic components of the geopotential, expected from new analyses of LAGEOS satellite data, can provide better constraints on the upper mantle structure.

A common feature of the whole set of models, is the decoupling effect of the chemical boundaries at 420 and $670 \mathrm{~km}$ portrayed by the two slowest relaxation modes at the bottom of each panel, the M1 and M2 modes respectively. The chemical layering induces large horizontal motions, as shown by the dark red and blue hues of these two modes, and the viscosity increase in the lower mantle acts to further enhance the horizontal deformation in the outer shells. The modes M1 and M2 carry more tangential deformation than radial one, as indicated by the darker colours in the right portions of the rings. This finding has important implications in the interpretation of the displacement fields induced by post-glacial rebound. The fact that the M1 and M2 modes carry essentially horizontal deformation means that chemical layering may not play a crucial role in the interpretation of vertical motion data but can be important for the analyses of horizontal intraplate deformation forced by the Pleistocene melting. This aspect will be analysed in deeper detail in a following set of calculations. Another consequence of this finding is that the horizontal displacements decay more slowly than the radial components.

The effects of a hard garnet layer, with a viscosity increase of a factor 50 with respect to the uppermost mantle, are visible for both the radial and horizontal deformations of the $l=2$ and $l=9$ components. In particular, we note the coupling effect of the hard layer in the circulation manifested in the upper branch solutions. This is portrayed by the reduction in the brightness of the white colour in the last two panels for the UB columns. The decoupling of the upper and lower mantle circulation due to the viscosity increase in the lower mantle is reduced by the increase of the mean viscosity in the upper mantle with garnet which becomes mechanically similar to the lower mantle and is thus dragged by the lower mantle flow. This effect is particularly important for the M1 and M2 modes of the UB and $l=2$ component.

\section{SIGNALS DUE TO THE $l=2$ HARMONIC COMPONENT: POLAR WANDER AND $\dot{j}_{2}$}

We can also estimate the effects of the hard layer on the perturbations of the Earth's rotation and on the time dependence of the Earth's flattening $j_{2}$, induced by the
Table 3. Properties of major ice sheets.

\begin{tabular}{|c|c|c|c|c|}
\hline Parameter & Symbol & Laurentide & Fennoscandia & Antartica \\
\hline Ice sheet mass & $\mathbf{M}$ & $1.8 \times 10^{19} \mathrm{~kg}$ & $6.0 \times 10^{18} \mathrm{~kg}$ & $5.4 \times 10^{18} \mathrm{~kg}$ \\
\hline $\begin{array}{c}\text { Angular radius } \\
\text { of ice sheet }\end{array}$ & $\alpha$ & $15^{\circ}$ & $10^{\circ}$ & $20^{\circ}$ \\
\hline $\begin{array}{l}\text { nitial displacement } \\
\text { of the center of the } \\
\text { ice sheet from the } \\
\text { axis of figure }\end{array}$ & $\theta$ & $25^{\circ}$ & $25^{\circ}$ & $0^{\circ}$ \\
\hline $\begin{array}{l}\text { Longitude of the } \\
\text { centroid }\end{array}$ & $\phi$ & $90^{\circ} \mathrm{W}$ & $20^{\circ} \mathrm{E}$ & $\ldots$ \\
\hline
\end{tabular}

ice-sheet disintegration. The source parameters are listed in Table 3 ; the time history in given by a sequence of $\mathbf{1 0}$ complete cycles of glaciation and deglaciation, with a loading phase of $9 \times 10^{4} \mathrm{yr}$ and an unloading one of $10^{4} \mathrm{yr}$. The results today are recorded $6 \times 10^{3} \mathrm{yr}$ after the end of the unloading phase. The effects of other source parameters, such as the time-dependent radii of the ice-sheets or a more realistic geographical distribution of the loads (Wolf 1987; Mitrovica \& Peltier 1989; Lambeck et al. 1990) are not considered in this work, where we are mainly concerned with the physics of the transition zone.

The complex formulation for the linearized set of Liouville equations, derived by Sabadini et al. (1984b) for a two-layer mantle, has been generalized to include the hard middle layer. In the Laplace transform domain, the excursions of the rotational axis with respect to the Earth's surface are properly described by

$\mathbf{m}(s)=\left(\frac{\mathbf{A}_{1}}{s}+\sum_{i=1}^{9} \frac{\mathbf{A}_{i+1}}{s-\mathbf{a}_{i}}\right) \frac{I_{x z}(s)+i I_{y z}(s)}{C-A}$

where $s$ denotes the Laplace variable. In this equation, $\mathbf{m}=m_{1}+i m_{2}$ where $m_{1}$ and $m_{2}$ are the director cosines of the axis of rotation in the geographical frame of reference. The difference between the polar and equatorial moments of inertia $C-A$ is related to the equatorial bulge. The term $I_{x z}(s)+i I_{y z}(s)$ represents the perturbations of the offdiagonal components of the inertia matrix, which are driving the polar displacement.

The set of complex residues $\mathbf{A}_{i}$ and inverse relaxation times $\mathbf{a}_{i}$ is derived from the solution of a $l=2$ tidal boundary value problem (Takeuchiet al. 1962). They arise from the interaction between the perturbed centrifugal potential and the induced mantle flow. The residue $\mathbf{A}_{1}$ accounts for the steady-state polar wander and it generalizes, for a stratified, viscoelastic Earth, the secular term used by Munk \& Macdonald (1960) to estimate the amount of polar drift driven by the distribution of the continents. The other complex residues $\mathbf{A}_{i+1}$ and their associated relaxation times $\mathbf{a}_{i}$ describe the transient contributions. They come into play on a short time-scale of a few thousand years following the end of deglaciation, while the $\mathbf{A}_{1}$ residue controls the 


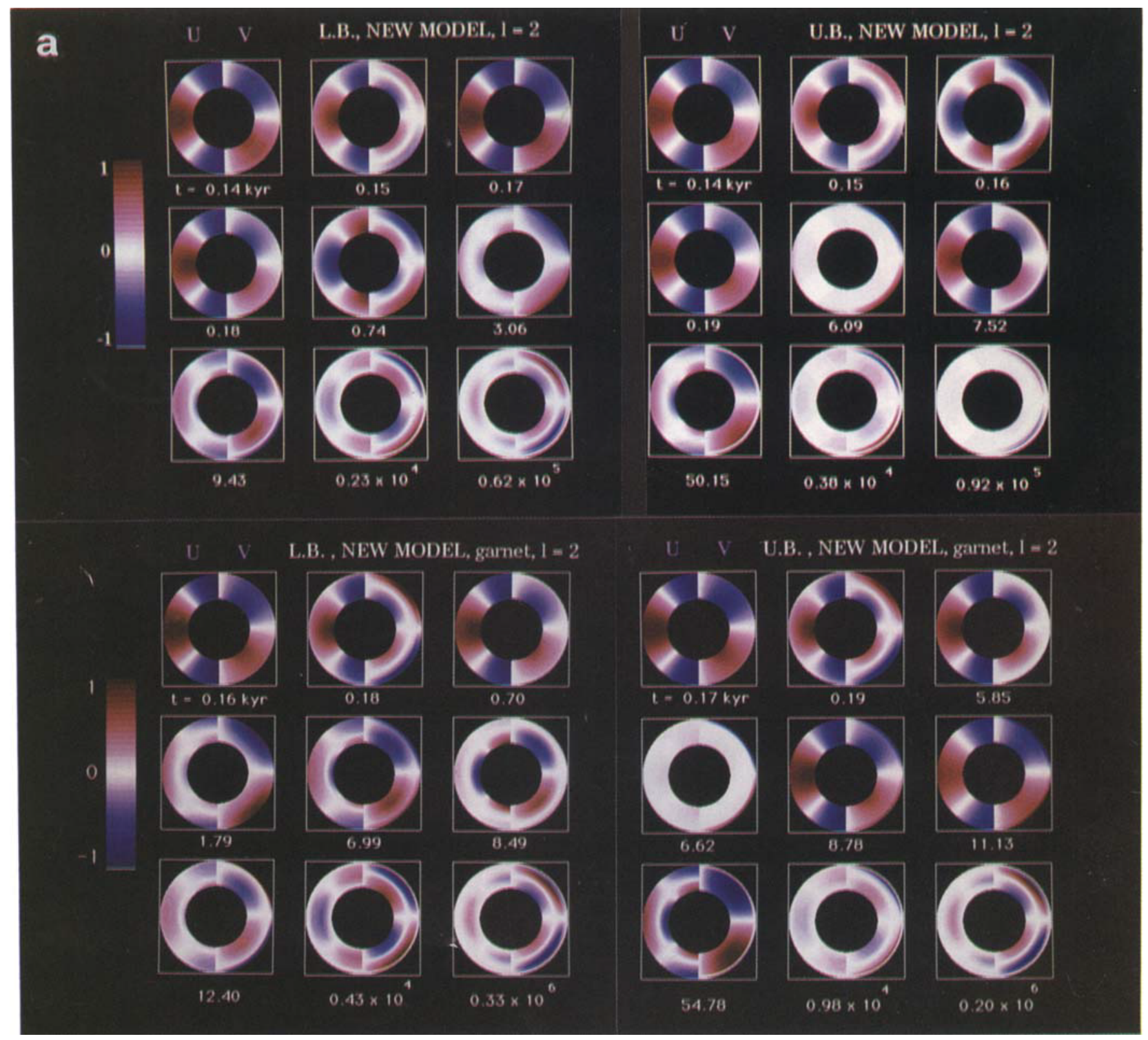

Figure 4. New model NM. For the $l=2$ and $l=9$ harmonic loading, (a) and (b) respectively, the radial and tangential components of the displacement for each of the nine modes carried by the five-layer model are portrayed. The modes are ordered as a function of their relaxation time in kyr. The left portion of the ring corresponds to the radial component $U$ while the right portion corresponds to the tangential component $V$. The amplitude of each mode is normalized to one. The colour palette has 25 evenly spaced intervals and is linear in magnitude. The lower and upper mantle viscosities are varied as shown in Table 2 for the lower and upper branches LB, UB respectively. In the first two panels on the top only the lower mantle viscosity is varied, while in the last two panels at the bottom, the effects of a viscosity increase in the hard layer with $C=50$ are considered. 


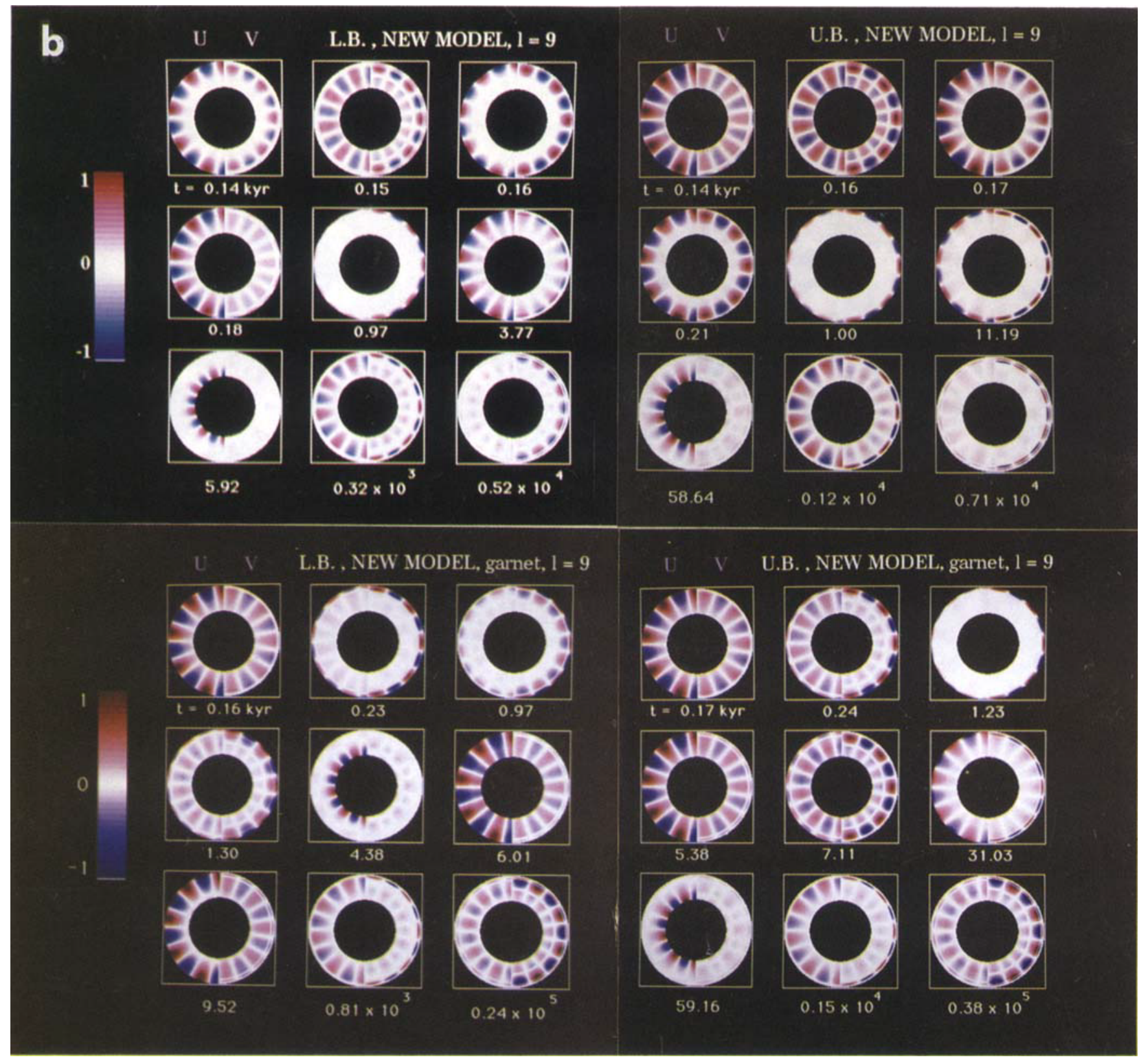

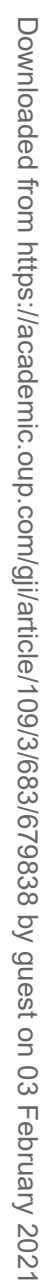




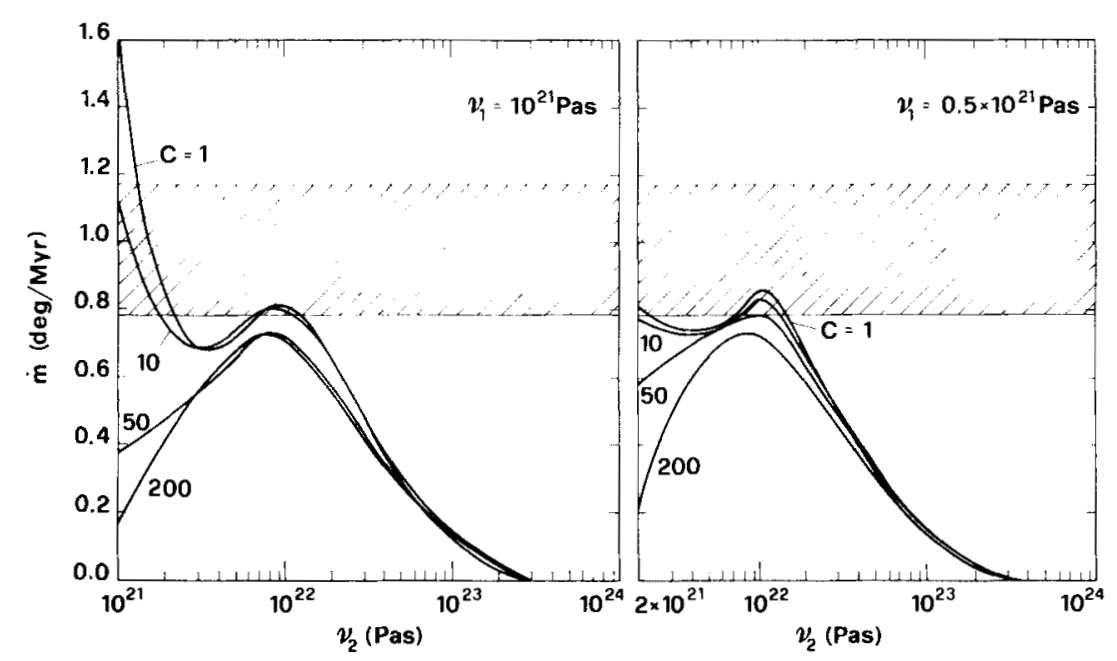

Figure 5. Effects of the hard layer on the rate of polar motion for varying lower mantle viscosity. The old and new models are given in the left and right panels respectively. Each curve corresponds to a different value of the parameter $C$, varied from 1 to 200 . The horizontal scale in the right panel begins with the lower mantle viscosity appropriate for the new model NM, lower branch.

rotation of the planet on time-scales of $10^{6}$ to $10^{7} \mathrm{yr}$ (Ricard \& Sabadini 1990).

The off-diagonal components of the inertia tensor $I_{x z}(s)+i I_{y z}(s)$ represent the perturbations due first, to the direct redistribution of surface loads and second, to the induced viscoelastic-elastic isostatic compensation. These two contributions tend to cancel each other. They can be easily quantified if we introduce the Love number $k_{2}^{\prime}(s)$ of degree 2 which accounts for the geopotential perturbation

$I_{x z}(s)+i I_{y z}(s)=\mathbf{G}(\theta, \phi)\left[1+k_{2}^{L}(s)\right] f(s)$,

where $\mathbf{G}(\theta, \phi)$ depends on the intensity and geometry of the loads located at the colatitude $\theta$ and longitude $\phi$ and where $f(s)$ is the Laplace transform of their time dependence. The constant 1 comes from the direct effect $(t=0)$ of the loads on the moment of inertia while the loading Love number $k_{2}^{L}$ accounts for the effects of isostatic compensation. The Love number $k_{2}^{L}$ depends upon the mechanical stratification of the model Earth and contains an elastic contribution $k_{e}$ plus a sequence of terms $k_{i}$ and their associated inverse relaxation times $s_{i}$

$k_{2}^{L}=k_{e}+\sum_{i=1}^{9} \frac{k_{i}}{\left(s-s_{i}\right)}$.

In Fig. 5 we show the speed of polar wander $\dot{\mathbf{m}}$ as a function of the lower mantle viscosity $v_{2}$. The upper mantle viscosities $v_{t}=10^{21}$ and $0.5 \times 10^{21} \mathrm{Pas}$, for the $\mathrm{OM}$ and the NM respectively, have been considered.

The observed polar wander with its uncertainty is taken from Lambeck (1980) and Dickman (1981) and ranges in the shaded band. As in the previous calculations, the parameter $C$ is varied from 1 to 200 . For a smooth increase in the viscosity of the hard layer $(C=10)$, we obtain a multiplicity in the lower mantle viscosity solutions, since we can fit the observed data for two widely spaced values, close to $10^{21}$ and $10^{22} \mathrm{Pas}$. For the two-layer Maxwell model of the mantle corresponding in our calculation to $C=1$, this multiplicity has been first pointed out by Yuen $e t$ al. (1986). The most striking result of these calculations is that a relevant viscosity increase in the hard layer $(C=50,200)$ breaks the multiplicity of the solution and we can barely fit the data for the new upper mantle viscosity model with $C=50$ (right). Even for this new model, a further viscosity increase in the hard layer drastically reduces the viscous flow in the mantle and inhibits the ability of the planet to wander. From these findings we demonstrate that polar wander data can be used to put bounds on the viscosity increase in the transition zone across the $420 \mathrm{~km}$ discontinuity.

In Fig. 6 we show the same sensitivity analysis for the temporal variations of $J_{2}$, for the old upper mantle model (left) and the new one (right). $j_{2}$ depends only on the time history of the loads and on the isostatic modes by a mathematical expression identical to the right-hand member of equation (11). The observed data are taken from Cheng et al. (1989). As for the case of $\dot{\mathbf{m}}$, we have a multiplicity in the solution. The data can be fit by a lower branch with $v_{2}$ ranging from $2 \times 10^{21}$ to $6 \times 10^{21} \mathrm{Pas}$ or by an upper branch close to $10^{23} \mathrm{Pas}$. The perturbation in the lower mantle viscosity is larger for the lower branches, and can deviate by a factor 3 from the reference model $C=1$. Perturbations are smoother for the upper branches, for both $v_{3}=10^{21}$ and $v_{1}=0.5 \times 10^{21}$ Pas. A high viscosity increase $(C=50,200)$ in the hard layer tends to diminish the gap between the solutions of the lower and upper branches. These results agree with the findings shown by Peltier (1985) who parametrized the effects of a thermally controlled viscosity increase in the transition zone by means of an internal lithosphere'.

In contrast to $\dot{m}$, the $j_{2}$ data are less diagnostic in retrieving the lower mantle viscosity because we cannot break the multiplicity of the two solutions, even with a high viscosity increase in the hard layer. This is due to the combined effects from both tidal and loading boundary conditions associated with the polar wander solutions that make the planet more sensitive to the presence of the hard layer in comparison with the purely mass loading boundary conditions for $j_{2}$. Temporal variations in the higher harmonic components of the geopotential are expected to be more diagnostic in the inversion of the upper mantle viscosity profile. 

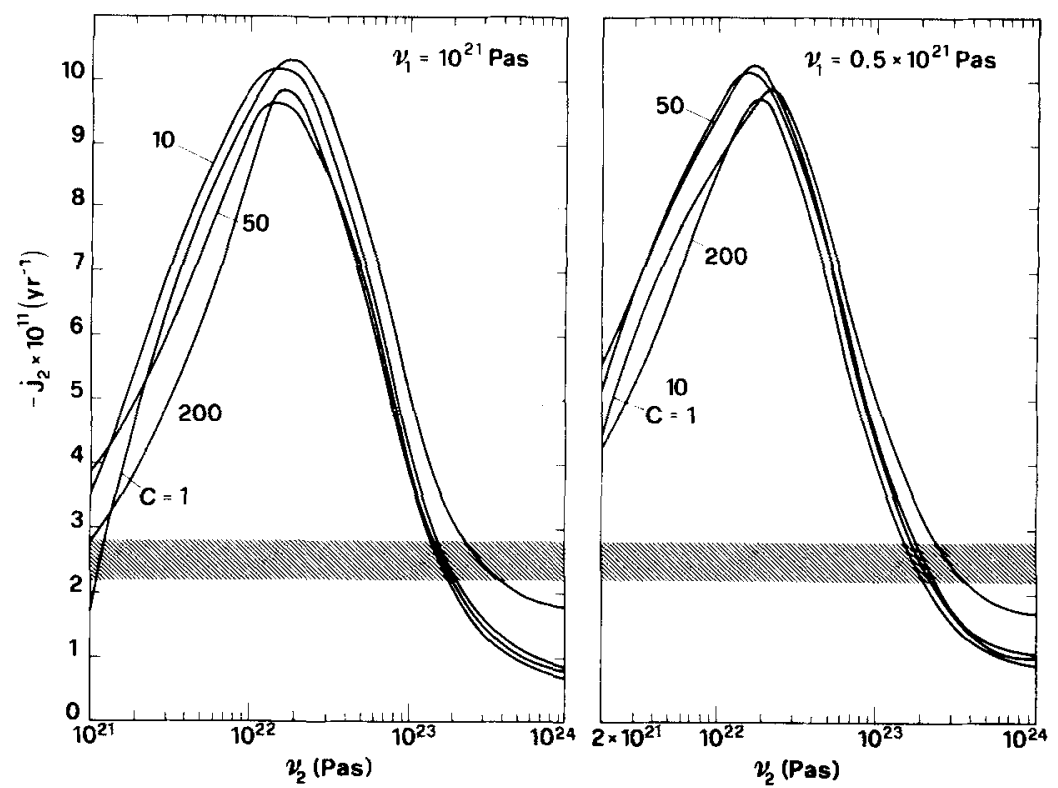

Figure 6. Effect of the hard layer on the temporal variation of the $l=2$ geopotential coefficient, in $\mathrm{yr}^{-1}$. Everything else is identical to Fig. 5 .

\section{FREE-AIR GRAVITY ANOMALIES VERTICAL AND HORIZONTAL DISPLA CEMENTS}

We study the sensitivity of the geophysical observables on the viscosity increase in the hard layer. We include 10 complete cycles and now on the loading phase, which lasts $10^{5} \mathrm{yr}$, is followed by an instantaneous unloading (Heaviside function). The results are thus recorded $1.2 \times 10^{4} \mathrm{yr}$ after the melting event. This is the same as recording the data at $7 \times 10^{3} \mathrm{yr}$ after the end of a deglaciation phase of $10^{4} \mathrm{yr}(\mathrm{Wu}$ \& Peltier 1982; Yuen et al. 1986). Fig. 7 deals with the negative free-air gravity anomaly at the centre of the Canadian shield. The expression given by Longman (1963) has been used to compute the gravity signal, while observed data are taken from $\mathrm{Wu} \&$ Peltier (1982). If the parameter $C$ is increased, viscosity solutions are moved to the left toward lower values. There is a trade-off between the viscosity of the hard layer and the viscosity of the lower mantle: an increase in the hard layer requires a simultaneous reduction in the lower mantle. In the left panel the data are not really discriminating on the lower mantle viscosity. This trade-off is more evident for the new upper mantle model in the right panel. The solution is more sensitive to the hard layer as a consequence of the softer upper mantle. In this case we obtain a perturbation in the possible lower mantle viscosity solutions by a factor 5 .

The effects of the hard layer on the time derivative of the free-air gravity anomalies, as a function of the angular distance from the centre of the Laurentide ice-sheet, are

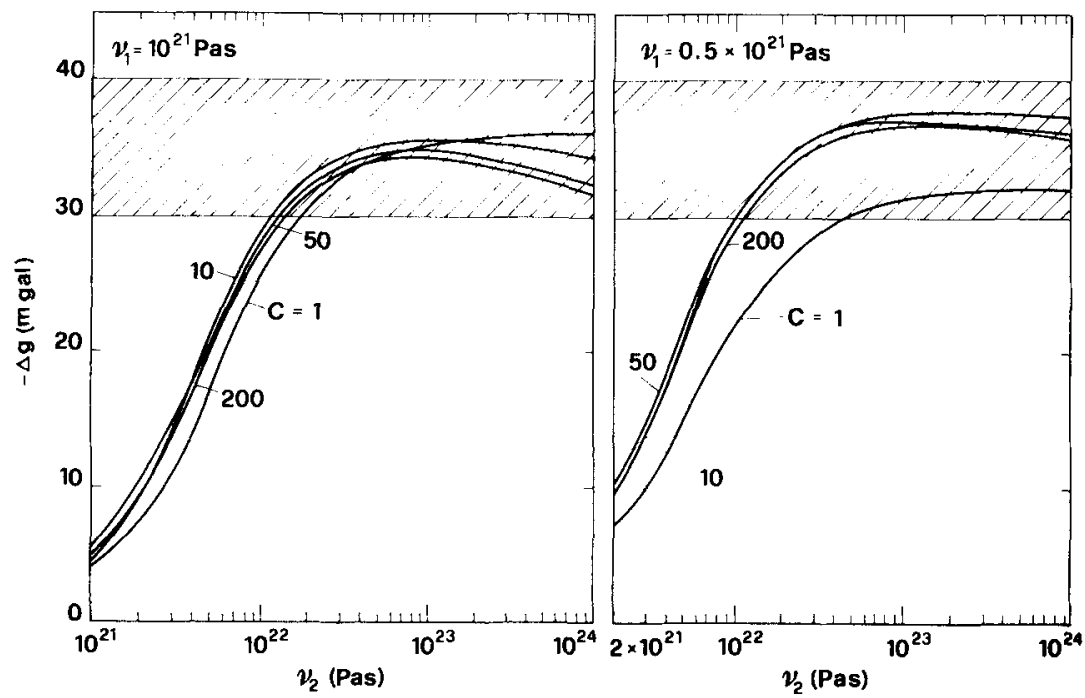

Figure 7. Effects of the hard layer on the free-air gravity anomaly at the centre of the deglaciated region in North America. Everything else is identical to Fig. 5. Henceforth, the ice-sheet disintegration is assumed to occur instantaneously and the observables are sampled at $12 \mathrm{kyr}$ after the melting event. 


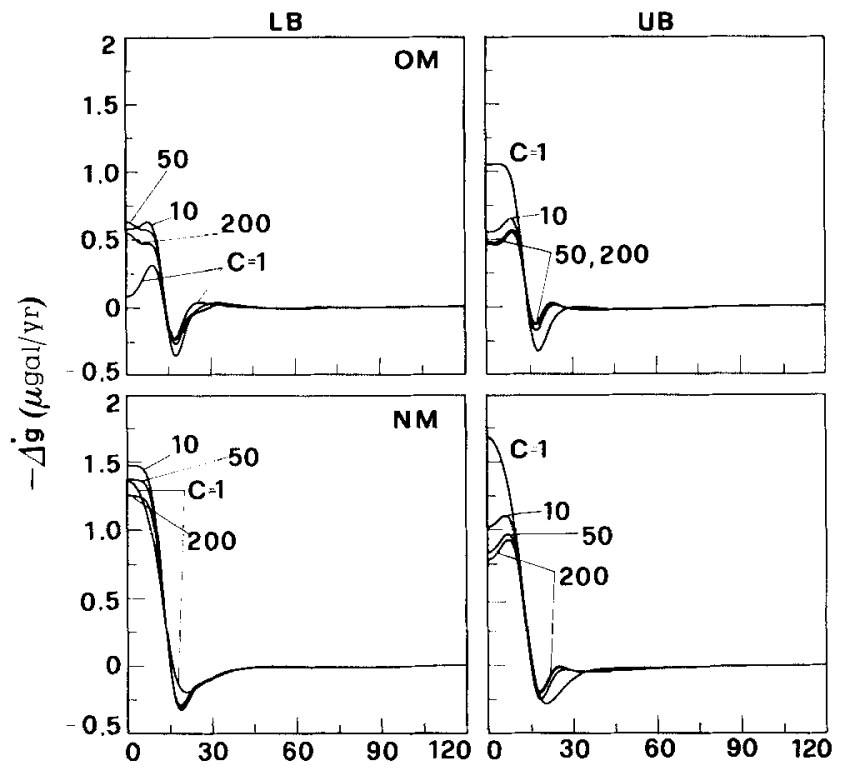

$\theta$ (deg)

Figure 8. Effects of the hard layer on the time derivative of the free-air gravity anomaly as a function of the angular distance from the centre of the deglaciated region in North America. Units are in $\mu \mathrm{gal} \mathrm{yr}^{-1}$. C is varied from 10 to 200 .

shown in Fig. 8 in $\mu$ gal $\mathrm{yr}^{-1}$. The reference models are the same as in Table 2 and the parameter $C$ is varied as shown in the panels. The signal in the centre can reach the largest anomaly of about $1.5 \mu \mathrm{gal} \mathrm{yr}^{-1}$. Except for the lower branch of the NM, the hardening of the transition zone drastically modifies the peak values in the centre by a factor of about 2 . A striking feature of these results is the sharpening of the signal in the load periphery for a viscosity increase in the hard layer. As it will be seen in Fig. 14, the findings of Fig. 8 closely resemble the pattern of the vertical velocity, thus suggesting that the free-air gravity anomalies are essentially controlled by the topography of the displaced surface boundary.

Fig. 9 deals with the effects of viscosity variations in the lower mantle on the surface vertical displacement $U$ (top) and its time derivative $\dot{U}$ (bottom), for the Laurentide ice-sheet and the old model OM. The left column stands for a thin lithosphere of $120 \mathrm{~km}$, whereas a thick lithosphere of $200 \mathrm{~km}$ is considered in the right column. The upper mantle is uniform $(C=1)$ and the ratio $B$ between the lower and upper mantle viscosities $v_{2} / v_{1}$ is varied from $B=1$ to $B=200$. As expected, in the top panels the central depression is larger for the high lower mantle viscosities than for the uniform models $(B=1)$, which is closer to the final configuration of equilibrium of a undeformed planet. The effects of lithospheric thickening (right panels) are minor for $B=10$ to 200 while for the uniform models the thick lithosphere acts to smooth the deformation both in the centre and periphery. The vertical velocity is more sensitive to viscosity variations in the lower mantle than the vertical displacements; this is portrayed by the large deviations, both in the centre and periphery, between the results corresponding to $B=10,50$ and 200 . It should be noted that

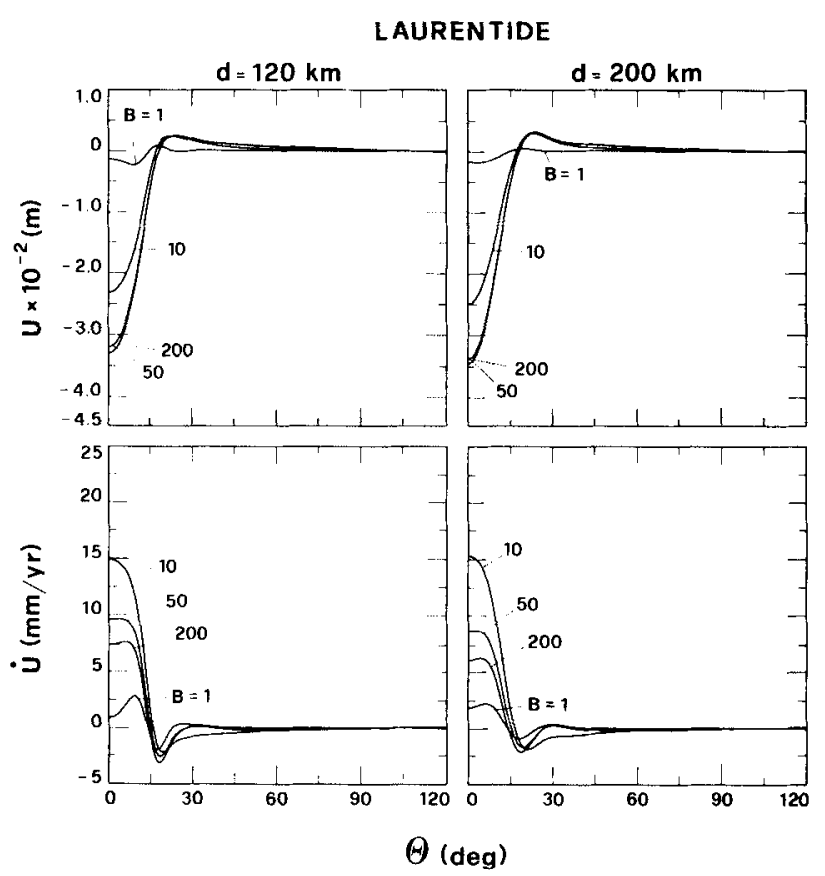

Figure 9. Effects of lower mantle viscosity variations on the vertical displacement in metres, top, and rare of vertical deformation in millimetres per year, bottom, for the Laurentide. Negative values of the displacements mean that the surface is depressed below the surface of the unperturbed Earth model; upward velocities are positive. The deformation is symmetrical around the left axis of each panel, corresponding to $\theta=0^{\circ}$. The lithospheric thickness $d$ is fixed at $120 \mathrm{~km}$ in the left column and to $200 \mathrm{~km}$ in the right one. The parameter $B$ is varied from 1 to 200 .

the behaviour of $\dot{U}$, resembles the sensitivity of $\dot{J}_{2}$ to viscosity increase in the lower mantle. A drastic enhancement in the vertical velocity for $B=10$ is followed by its reduction for a further viscosity increase in the lower mantle.

A similar analysis is performed in Fig. 10 for the rebound of the Fennoscandia ice-sheet. The difference between the Canadian and Fennoscandia ice-sheets arises from their difference in sizes (see Table 3). As the lithosphere in the Baltic region is generally thinner than under the Canadian shield (Calcagnile 1982; Grand 1987), we performed our computation for two lithospheric thicknesses $d=100 \mathrm{~km}$ and $d=150 \mathrm{~km}$, somewhat smaller than those used in Fig. 9. For the Fennoscandia rebound, the thinner lithosphere of $100 \mathrm{~km}$ must be preferred after Lambeck et al. (1990). It should be noted that the uniform model $(B=1)$ predicts larger vertical displacements and velocities than those induced by the response to the Laurentian ice-sheet. This is consistent with the findings of Fig. 9 which indicate that a viscosity increase in the lower mantle is required in order to fit the vertical velocity of at least $10-15 \mathrm{~mm} \mathrm{yr}^{-1}$ in the centre of the Laurentide ice-sheet (Walcott 1972).

The effects of a viscosity increase in the lower mantie on the horizontal displacement and its time-derivative are shown in Figs 11 and 12 for the Laurentide and Fennoscandia ice-sheets and old model OM. Similarly to what has been observed for the vertical components, a non-uniform mantle maintains a large isostatic disequilibrium even at $1.2 \times 10^{4} \mathrm{yr}$ after the ice-sheet disintegration 


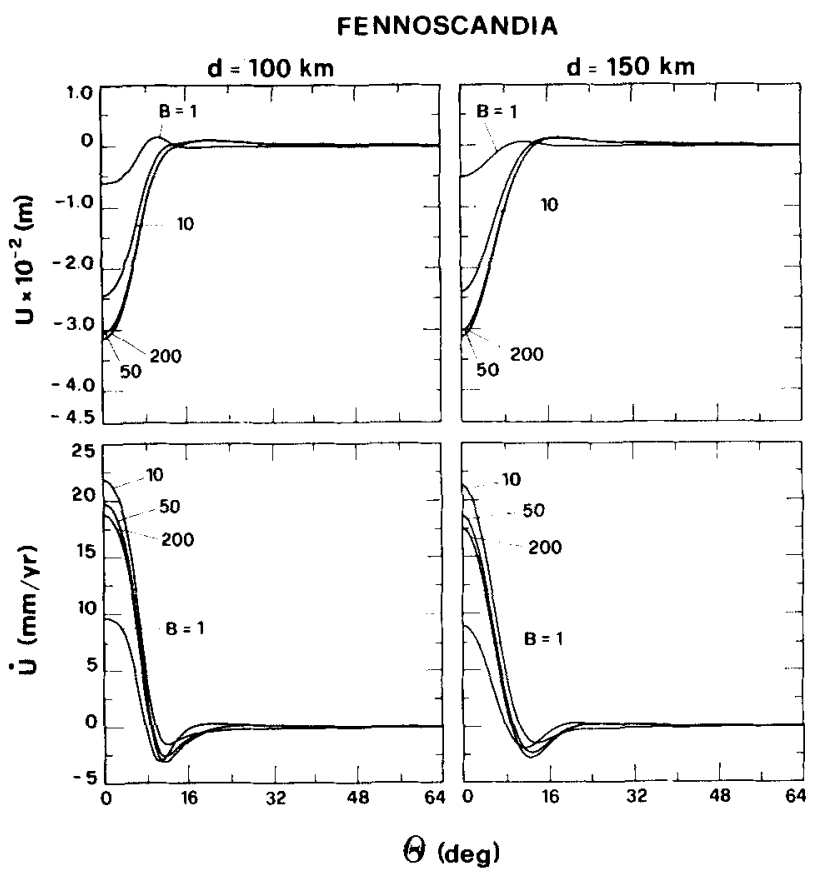

Figure 10. The same as in Fig. 9, except for the Fennoscandia ice-sheet with $d=100$ and $150 \mathrm{~km}$. Note that the horizontal scale of the distance has been modified.

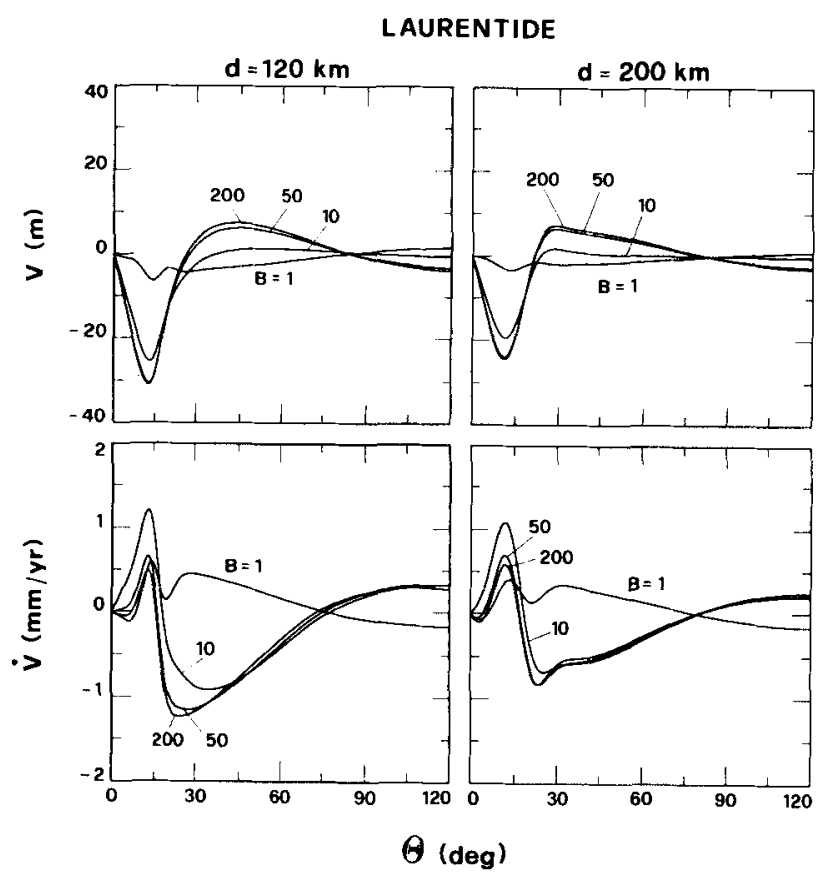

Figure 11. Effects of lower mantle viscosity variations on the horizontal displacement in metres, top, and rate of horizontal deformation in millimetres per year, bottom. Positive values are relative to displacements and velocities away from the centre of the ice-load. Viscosity variations in the lower mantle are the same as in Fig. 9. As in the previous figures, the deformation pattern is symmetrical around the left axis of each panel. The lithospheric thickness is the same as in Fig. 9.

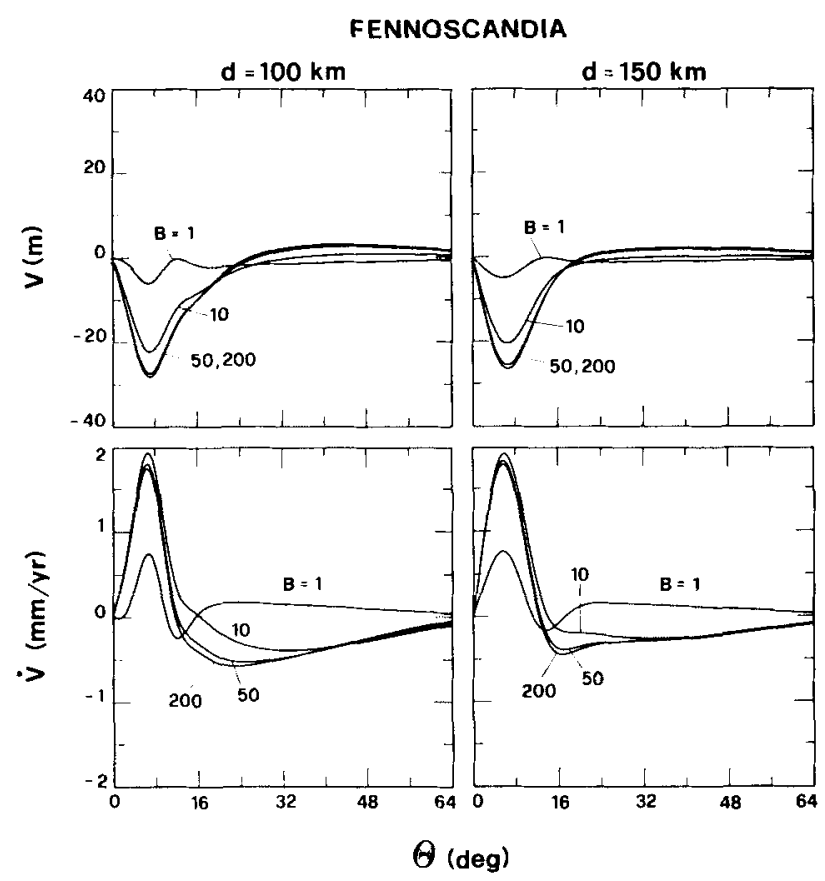

Figure 12. The same as in Fig. 11, except for the Fennoscandia ice-sheet with lithospheric thickness $d$ of 100 and $150 \mathrm{~km}$.

and induces large horizontal velocities and displacements both in the central and peripheral regions. For $B=10,50$ or 200 , the largest gradients are located in the proximity of the ice edge. A remarkable difference between vertical and horizontal motions is the slower decay with distance from the ice centre for the horizontal components. This can be easily understood from inspection of Figs 4(a) and (b) which show that the eigenfunctions associated with vertical deformation have much shorter characteristic times than those producing horizontal motions. This suggests that it is possible to detect this long distance deformation by means of VLBI techniques (James \& Morgan 1990).

The sensitivity of these results shows that horizontal motions can be used to put bounds on the viscosity increase in the lower mantle. For both thin and thick lithospheres, the largest horizontal displacements are concentrated at sites close to the ice edge. It should be noted that a viscosity increase in the lower mantle by one order of magnitude drastically increases the signal in the proximity of the ice edge and peripheral regions. Increasing the lower mantle viscosity further $(B=50$ to 200$)$ produces only minor modifications in the centre while large deviations from the models with $B=1$ to 10 are obtained in the periphery $\left(\theta>30^{\circ}\right)$. The horizontal velocities portray a slightly more complicated pattern and two asymptotic situations for the near-field $\left(\theta<15^{\circ}\right)$ and far-field $\left(\theta>15^{\circ}\right)$ are reached for $B=10$ and $B=50$ respectively. The substantial difference between the horizontal velocities induced by rebound in North America and Northern Europe is demonstrated by the larger positive values for the smaller ice sheet. The dominance of negative horizontal velocities for the Laurentide ice sheet is due to the huge amount of upper mantle material which is presently flowing toward the centre of the formerly glaciated area, after having been displaced outward during the glaciation phase. Our results clearly 

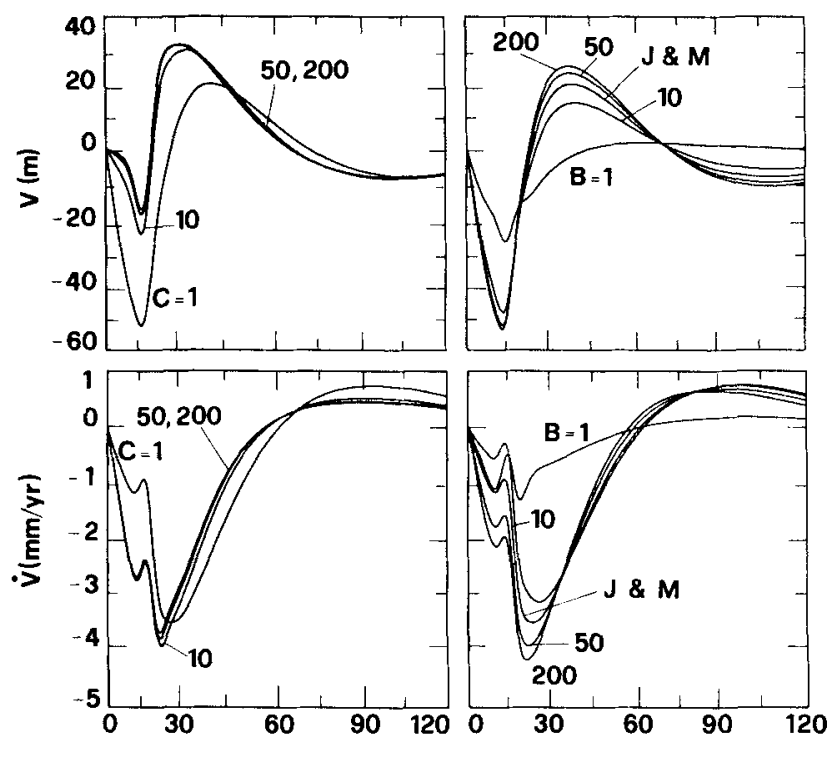

$\Theta$ (deg)

Figure 13. Comparison with the results of James \& Morgan (1990), for the horizontal displacement and rate of horizontal deformation. The model parameters are listed in Table 1 of James and Morgan's paper. They are: $d=120 \mathrm{~km}, \rho_{L}=2900 \mathrm{~kg} \mathrm{~m}^{-3}, \mu_{L}=4 \times$ $10^{10} \mathrm{~N} \mathrm{~m}^{-2}, \rho_{1}=3550 \mathrm{~kg} \mathrm{~m}^{-3}, \mu_{1}=7.1 \times 10^{10} \mathrm{~N} \mathrm{~m}^{-2}, v_{1}=10^{21} \mathrm{~Pa} \mathrm{~s}$, $\rho_{3}=3800 \mathrm{~kg} \mathrm{~m}^{-3}, \quad \mu_{3}=1.45 \times 10^{11} \mathrm{~N} \mathrm{~m}^{-2}, \quad v_{3}=10^{21} \mathrm{~Pa} \mathrm{~s}, \quad \rho_{2}=$ $4900 \mathrm{~kg} \mathrm{~m}^{-3}, \mu_{2}=4 \times 10^{11} \mathrm{~N} \mathrm{~m}^{-2}, v_{2}=2 \times 10^{22} \mathrm{~Pa} \mathrm{~s}$. The density of the core is $\rho_{C}=11100 \mathrm{~kg} \mathrm{~m}^{-3}$.

show a trade-off between the size of the surface load and the extent of the horizontal motions. The largest horizontal rate of strain, which is the difference between maximum and minimum velocities, is of the order of $2.5 \mathrm{~mm} \mathrm{yr}^{-1}$. This value is slightly smaller than the $4 \mathrm{~mm} \mathrm{yr}^{-1}$ found by James \& Morgan (1990) from a similar Earth model. It should be pointed out, on the other hand, that the horizontal motions are extremely sensitive to the elastic and density structure of the upper portion of the planet. As a benchmark calculation, we made use in our model of the same parameters listed by James \& Morgan (1990), referenced from now on as $J \& M$, in the reference model given in their Table 1. We should emphasize that the core in J\&M is characterized by an extremely low viscosity in order to simulate an inviscid core, as considered in our model. The results shown in Fig. 13, denoted by $\mathrm{J} \& \mathrm{M}$, are essentially identical to the ones portrayed in their Fig. 4 for the reference model. The minor deviations are due to the slightly different loading history. In our approach we preferred to constrain the total amount of chemical stratification in the mantle to 9 per cent, in agreement with seismological observations, while James \& Morgan (1990) favoured the idea of averaging the density stratification of the standard PREM model by Dziewonski \& Anderson (1981) in their three-layer mantle, thus overestimating the intensity of the non-adiabatic density discontinuities at the various interfaces. The differences between the right column of Fig. 13 and the left column of Fig. 11 can thus be interpreted as being due to effects from non-adiabatic density jumps. Our findings suggests that non-adiabaticity in the mantle favours large horizontal motions, in agreement with the pattern of modal deformations shown in Fig. 4. The larger restoring forces acting at a non-adiabatic density jump produce a channel-like effect in the upper mantle which enhances the horizontal motions (Sabadini \& Yuen 1989). As already observed in Fig. 4, the horizontal deformation shows a slower decay with time because it is dominant in the slowest $\mathrm{M} 1$ and $\mathrm{M} 2$ modes with respect to the radial component. The left column shows the effects of the hard layer in the presence of very high chemical discontinuities.

We now show the effects of the hard layer and lithospheric thickness variation on the vertical displacements and velocities, portrayed in the top and bottom panels of Fig. 14 for the Laurentide ice-sheet. The left column corresponds to the thin lithosphere of $120 \mathrm{~km}$, while the right one corresponds to a lithosphere of $200 \mathrm{~km}$ with the reference models of Table 2 .

The parameter $C$ is varied from 1 to 200 . Except for the displacements corresponding to the upper branch of the old model OM with $d=200 \mathrm{~km}$, all the calculations are extremely sensitive to viscosity increase in the transition zone both in the centre and in the periphery of the load. In particular, it should be noted that a viscosity increase in the hard layer is responsible for the steepening of the curves at the ice edge and, as expected, the effects are larger for the lower value $v_{1}=5 \times 10^{20} \mathrm{Pas}(\mathrm{NM})$. For the smooth viscosity increase in the lower mantle (LB) the hard layer acts to increase the rates of subsidence at the periphery. This subsidence, associated with the short-wavelength feature of the peripheral bulge, is highly sensitive to viscosity variations in the hard layer and the associated observation data can be used to resolve the upper mantle viscosity structure. If the parameter $C$ is increased beyond the threshold of 50 , all the different models predict an asymptotic configuration which shows minor modifications for an additional viscosity increase. The lithospheric thickening in the right column produces minor effects, with weaker deformation both in the centre and periphery.

In Fig. 15 the same analysis is performed for the horizontal displacements and velocities. In each panel, the viscosity and lithospheric thickness is identical to the corresponding panel of Fig. 14. At a first glance, variations in the parameter $C$ drastically modify the horizontal motion pattern, generally increasing the intensity of the gradients in the whole spatial domain, both in the near-field and in the far-field with respect to the former ice load. The presence of the high-viscosity region with $C>1$ is responsible for a delay in the displacement of upper mantle material toward the centre of the formerly glaciated area, as illustrated by the higher horizontal velocities toward Hudson Bay (bottom). We also observe here the tendency to reach an asymptotic configuration for $C \geqslant 50$. The thickening of the lithosphere from the left to the right column has the effect of reducing the difference between the largest positive and negative values in the displacements or velocities, reducing the amplitude of the deformation. The effects of lithospheric thickness variations are in any case minor compared to the effects due to viscosity variations in the hard layer.

The effects of the hard layer on the rebound in Fennoscandia are shown in Fig. 16 for a lithospheric thickness fixed at $100 \mathrm{~km}$. The vertical displacements and velocities are shown in the top panels while at the bottom we portray the horizontal components. The observations 


\section{LAURENTIDE}
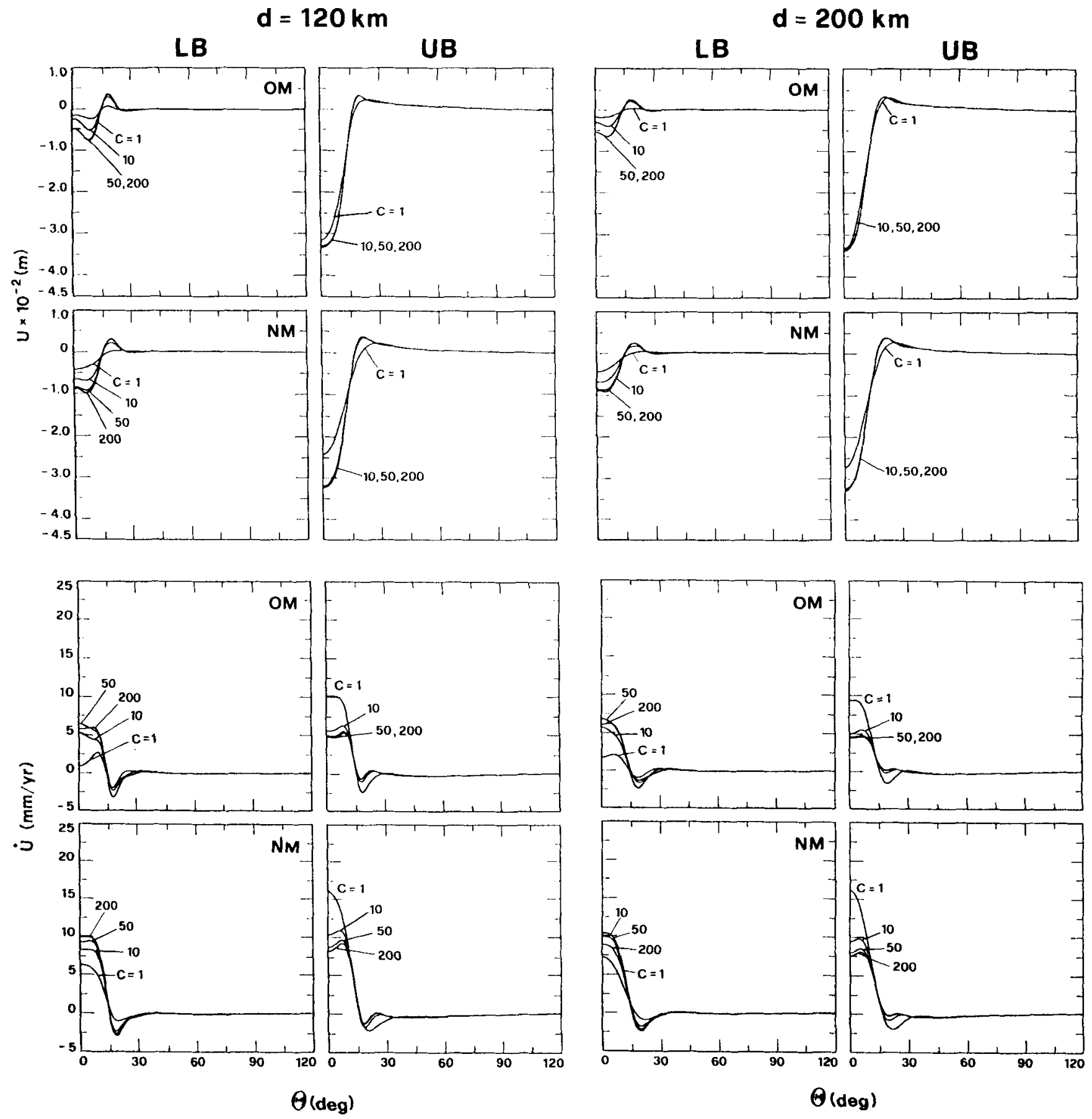

Figure 14. Effects of the hard layer on the vertical displacement, top, and rate of vertical deformation, bottom, induced by the Laurentide ice-sheet. The lithospheric thickness is the same as in previous figures, and the different models OM or NM and branches of the solutions LB or UB are arranged as in Table 2 . The parameter $C$ ranges from 1 to 200 .

already made for the Figs 14 and 15 are valid also for these calculations. Otherwise, we must note the generally larger positive horizontal velocities for Fennoscandia. This substantial deviation from the predictions of Fig. 15 for the rebound in North America is due to the smaller amount of mantle material being dragged toward the centre of the deglaciated area beneath Fennoscandia by the smaller ice-sheet. For $C>1$ we sharpen both the vertical and horizontal deformation in the peripheral region.

\section{STRESS FIELDS IN THE LITHOSPHERE AND UPPER MANTLE}

Figs 17 and 18 portray the temporal evolution of the maximum shear stress, defined as the difference between the maximum and minimum eigenvalues of the stress tensor, in a vertical half-cross section through the axis of symmetry, located at the left-hand side in each panel. The stress pattern is recorded at the instant of melting $t=0$, at $t=6$ 


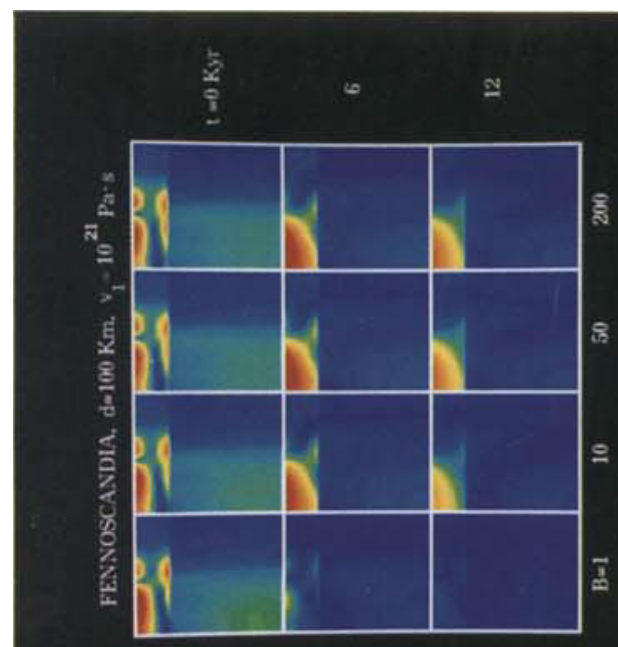

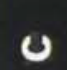

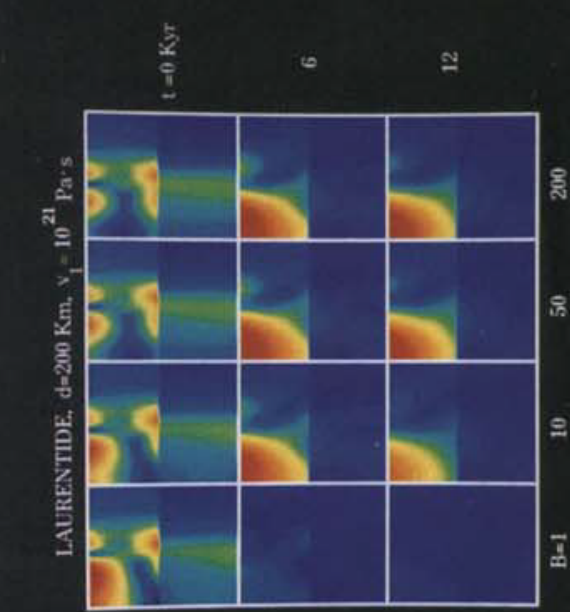

อ
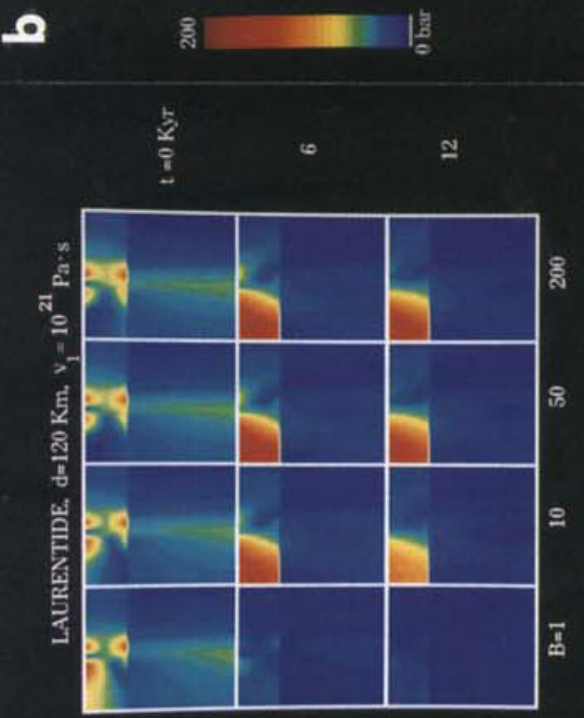

๘
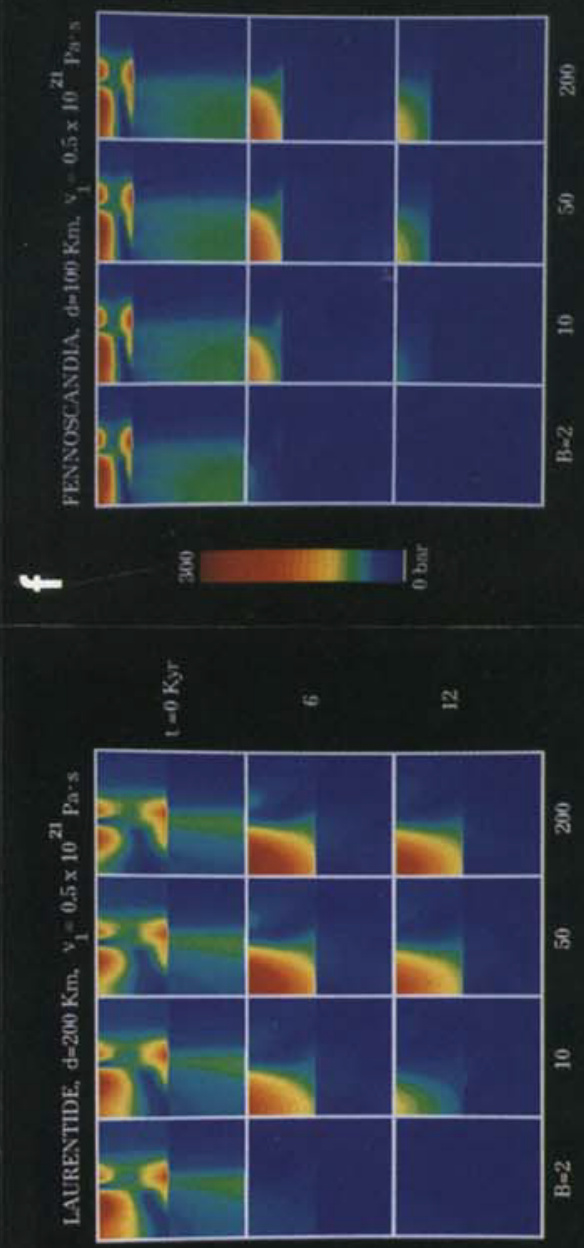

(1)

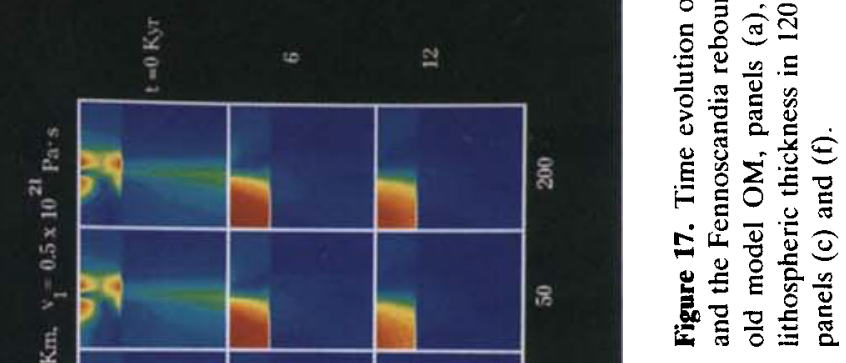

(อ)号它

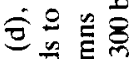

อे

(3)

要

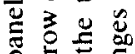

온 준

它

당

踪可

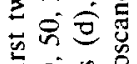

원

只苋记

正安

可的艺

8 원

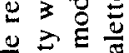

늘

可㣢

承

苋

당

공 동

然

릉

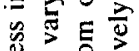
战总 司造 的证

E

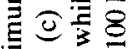
的它 월웡

을

워

은

훙

炡

응잉

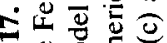

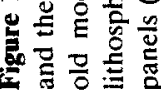




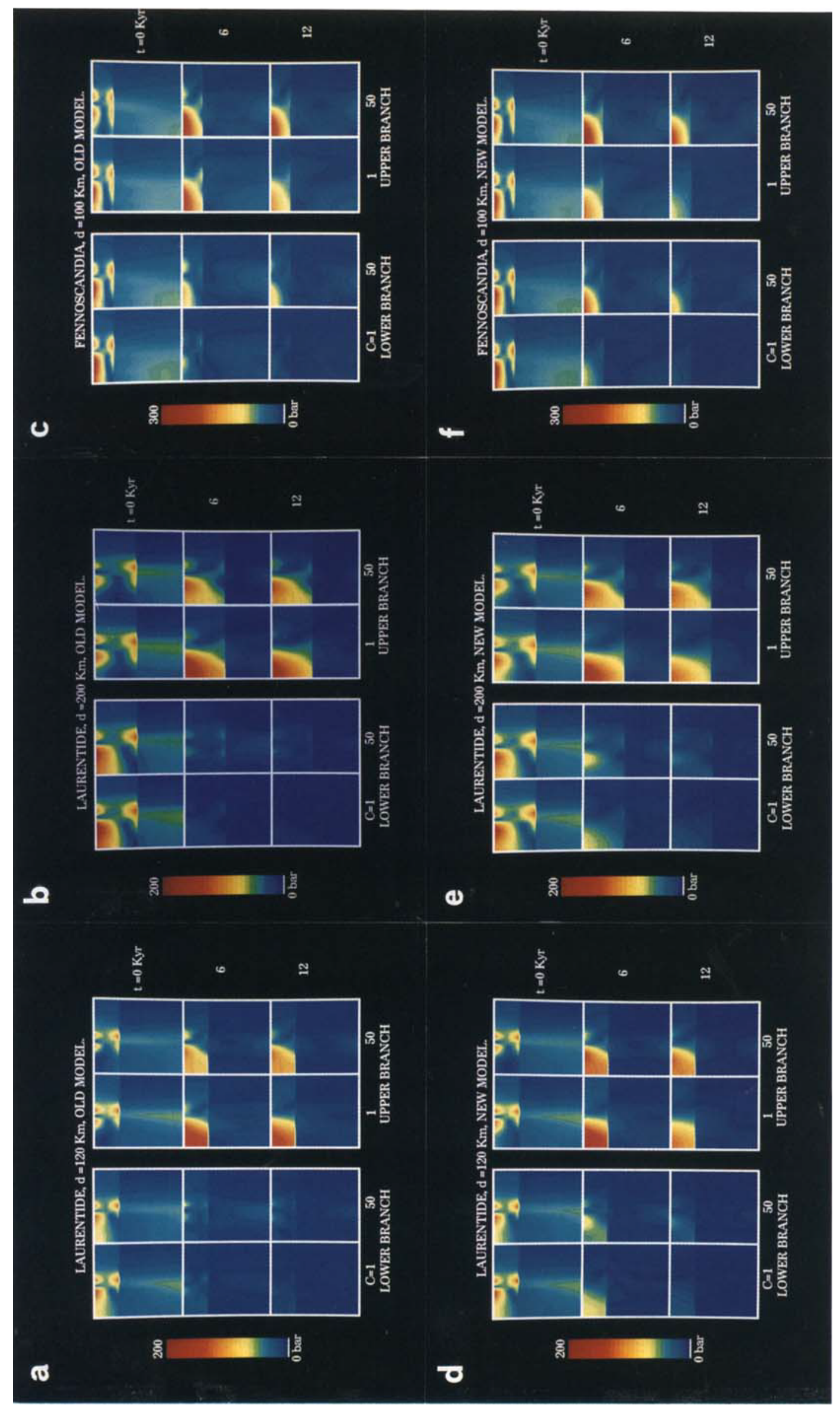


a

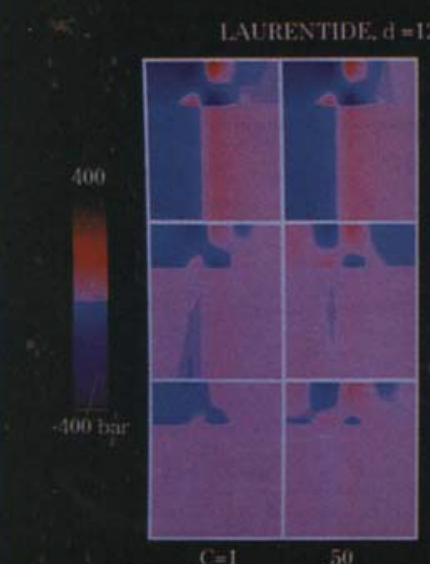

LOWER BRANCH

C

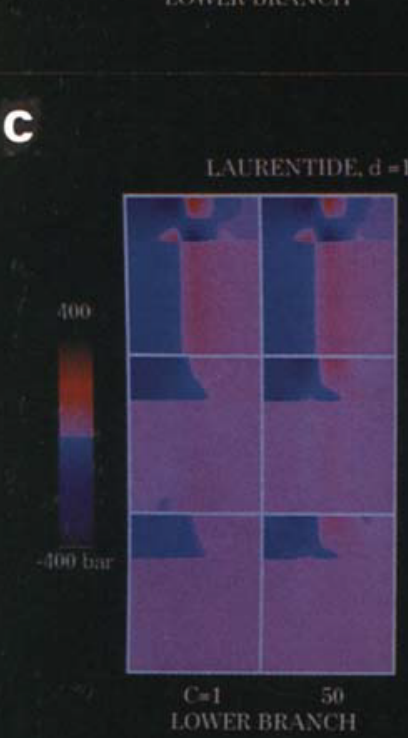

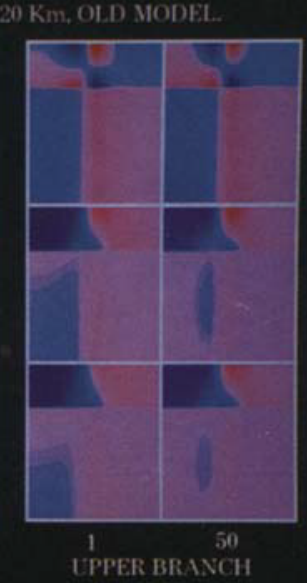

LPPER BRANCH

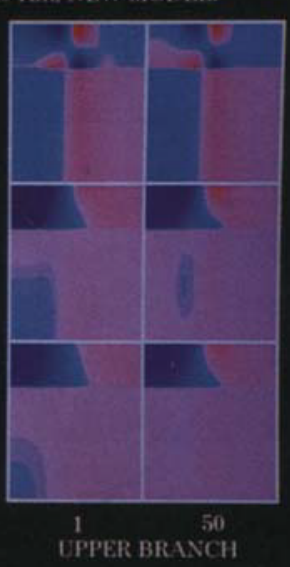

b

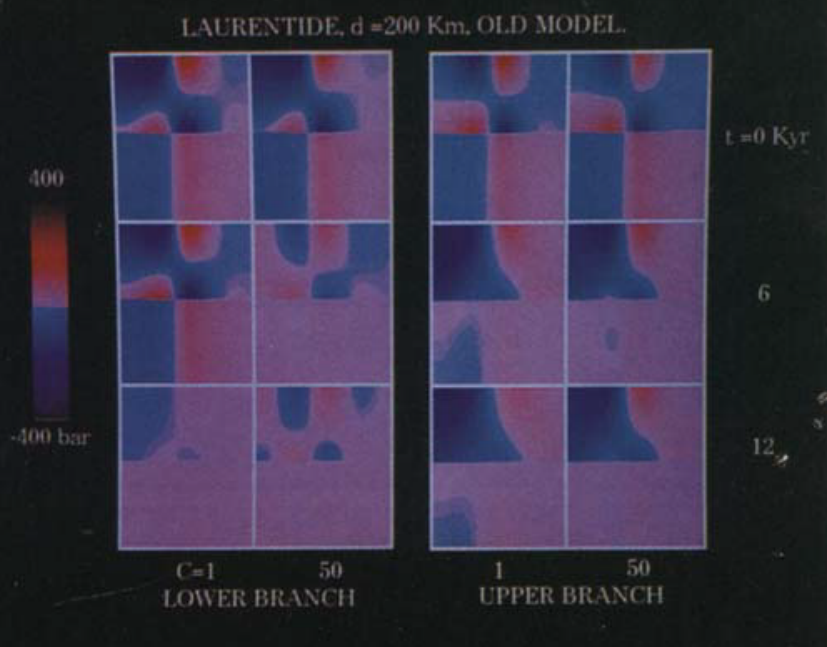

d

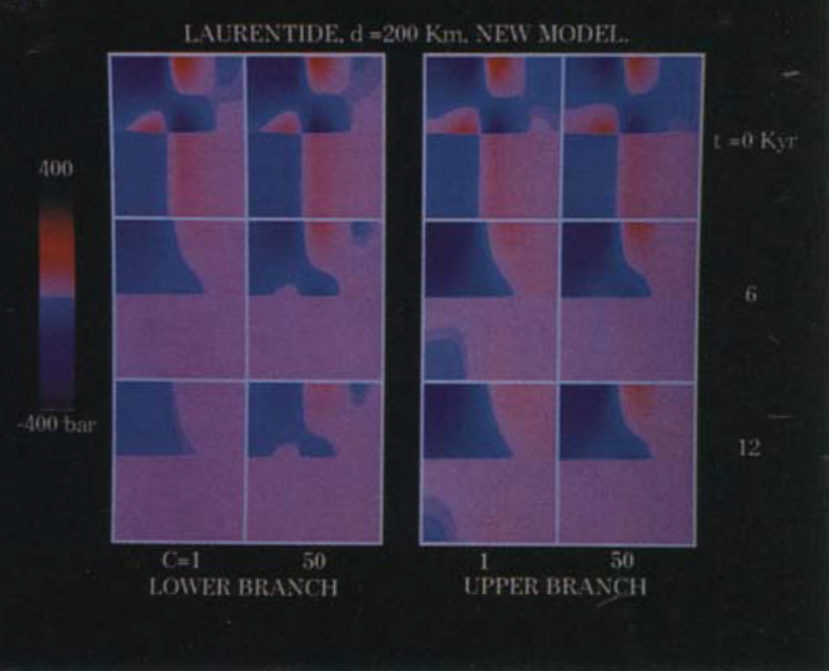

Figure 19. State of tension or compression given by $f(\theta, r)=\sigma_{\theta H}-\sigma_{r r}$ for the Laurentide ice-sheet, with $d=120 \mathrm{~km}($ left) and $200 \mathrm{~km}$ (right) The upper and lower branch, with $C=1$ and $C=50$ are shown for the old model OM (top) and the new model NM (bottom).

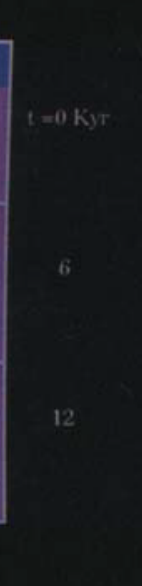




\section{LAURENTIDE}

$\mathrm{d}=120 \mathrm{~km}$

LB
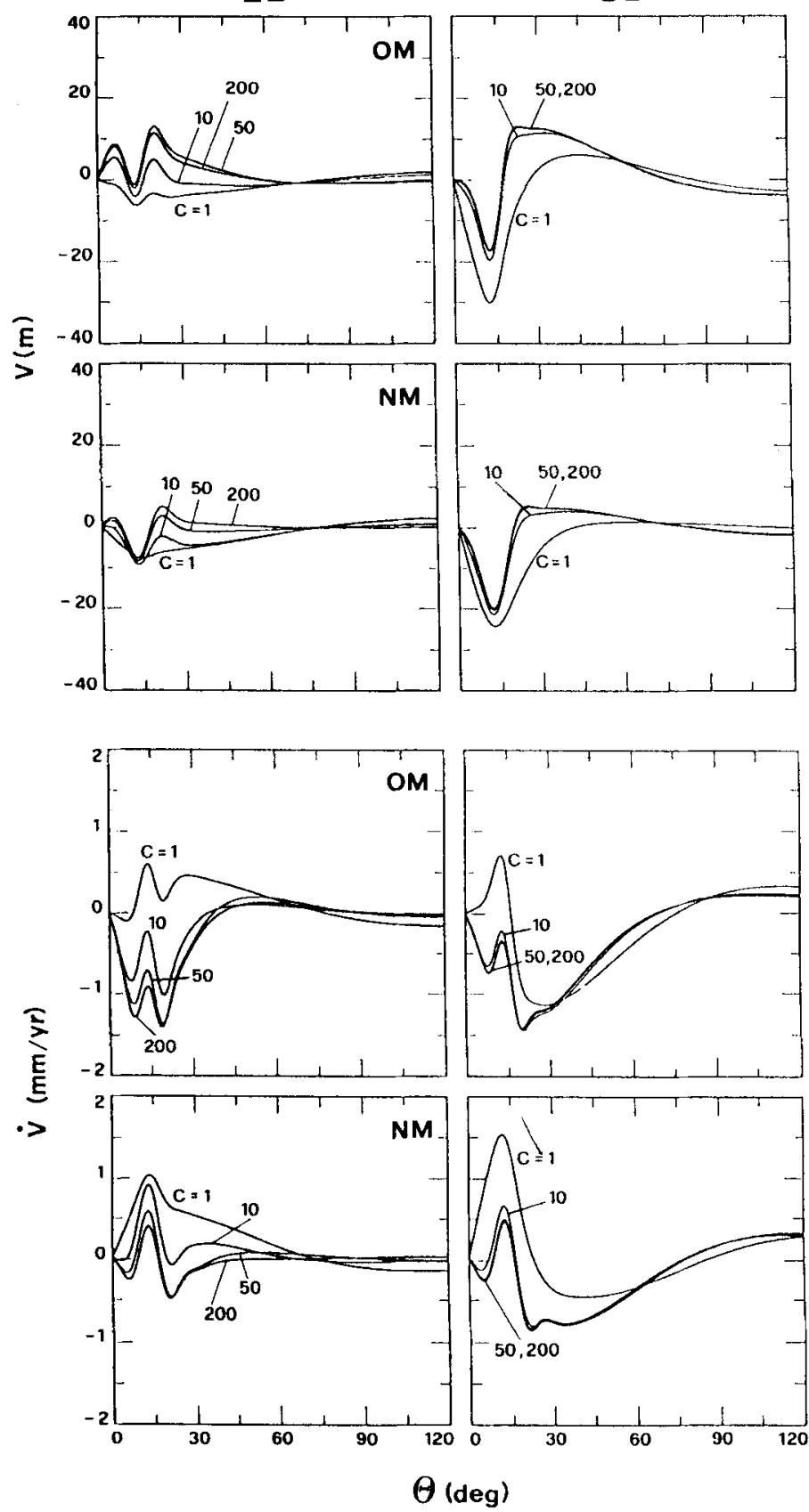

$\mathrm{d}=200 \mathrm{~km}$

LB UB
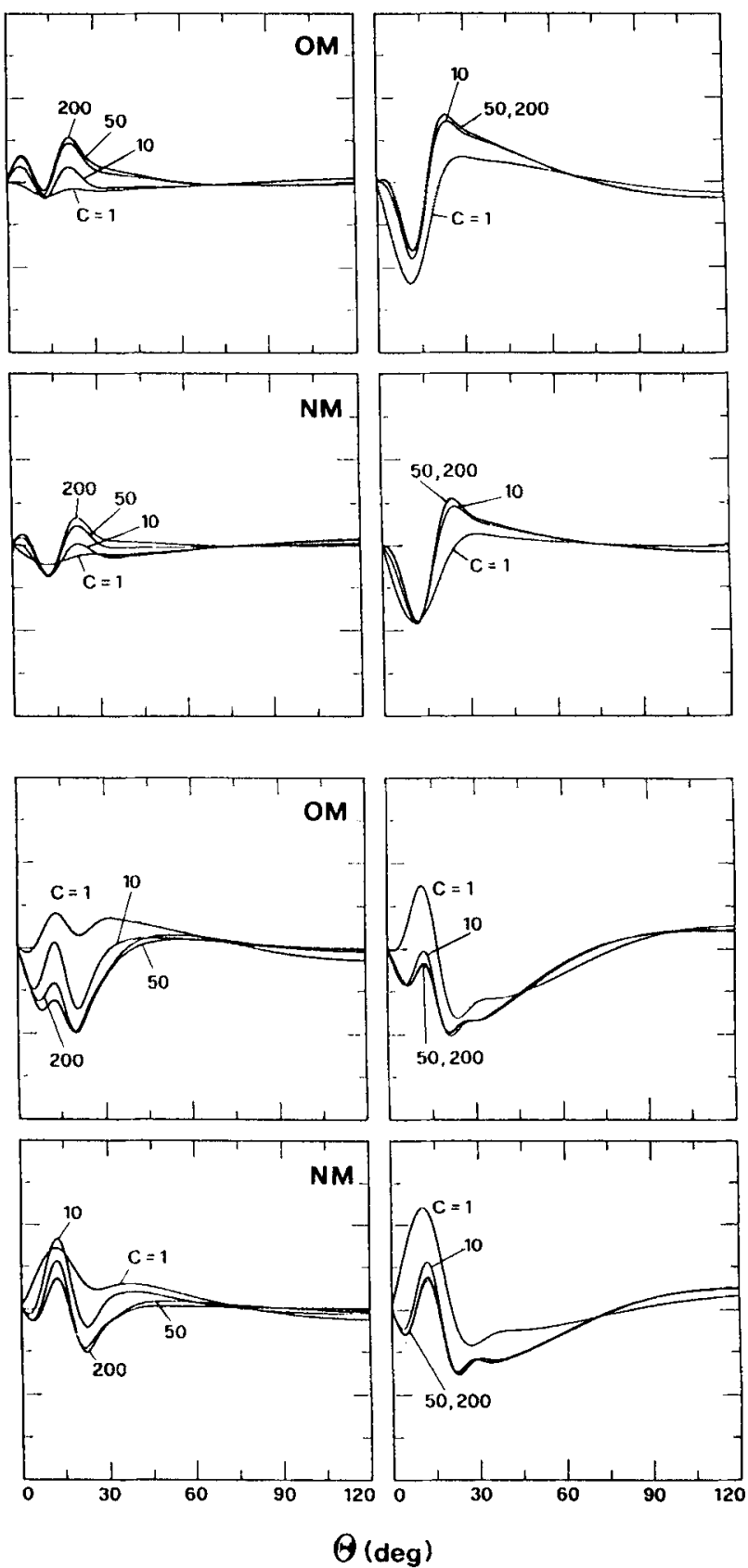

Figure 15. As in Fig. 14, except for the horizontal component of the deformation.

and $12 \mathrm{kyr}$, which corresponds to the present time. The top and bottom rows stand for the old (OM) and new (NM) upper mantle viscosity models, the first two columns on the left correspond to the Laurentide ice-sheet with lithospheric thickness $d$ of 120 and $200 \mathrm{~km}$ and the third column corresponds to the results for the Fennoscandia ice-sheet. In Fig. 17, the lower mantle viscosity is varied, as given by $B$ at the bottom of each panel. The discontinuity of the maximum shear stress, highlighted by the colour contrast at the lithosphere-upper mantle interface, is due to its dependence on the components $\sigma_{\phi \phi}, \sigma_{\theta \theta}$ that do not enter the continuity equations and to the rheological contrast.

The most striking result of these calculations is the disappearance of the maximum shear stress, both in the lithosphere and upper mantle, for the uniform models $(B=1)$, or mild viscosity contrasts $(B=2)$, at $t=12 \mathrm{kyr}$. This is in distinct contrast with the findings for $B \geq 10$, that show the focusing of the stress in the lithosphere due to the delayed upward migration from the stiff lower mantle (Spada et al. 1991b). For longer times, the stress is relaxed 
FENNOSCANDIA, $d=100 \mathrm{~km}$
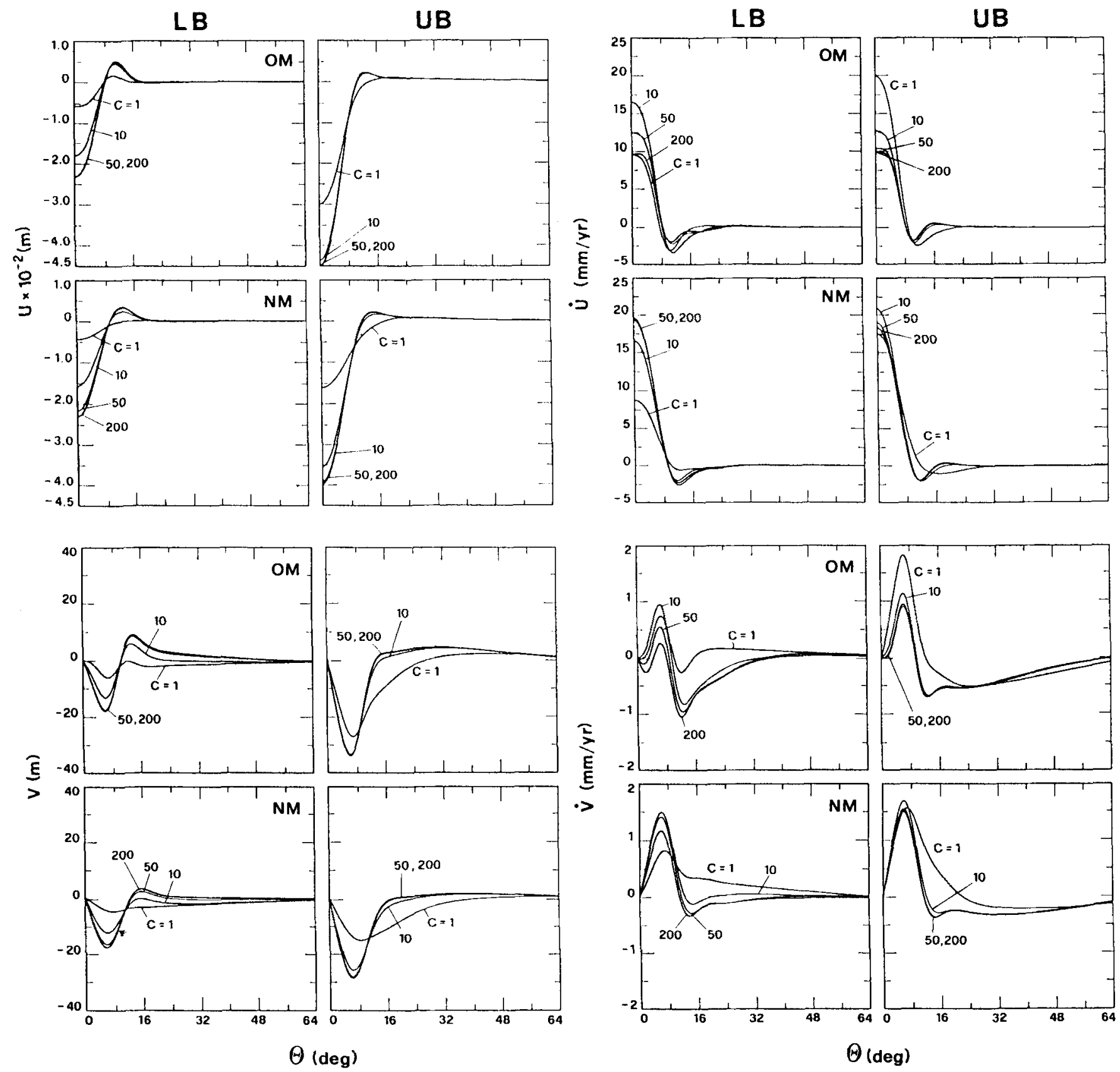

Figure 16. Effects of the hard layer on the vertical and horizontal displacements and their time derivatives induced by the Fennoscandia rebound. Model parameters are the same as in previous figures, except for the lithospheric thickness $d$ fixed at $100 \mathrm{~km}$.

in the whole planet, as for the uniform viscosity model. For $B \geq 50$, the maximum shear stress in the lithosphere can be as high as 100 bar and pre-existing faults induced by a previous tectonics, such as the opening of the NorthAtlantic, can be reactivated by the unloading event (Stein $e t$ al. 1979; Hasegawa \& Hermann 1989; Bungum 1989). These results indicate that the existence of an active seismicity around deglaciated areas requires a substantial viscosity increase in the lower mantle, in agreement with recent findings from Holocene rebound data and sea-level fluctuations in NW Europe (Lambeck et al. 1990). The recorded seismicity along passive continental margins can thus become an useful tool to put bounds on the viscosity profile especially in the lower mantle (Spada et al. 1991b) and must be added to other geophysical observables associated with post-glacial rebound or geoid anomalies due to the internal mass distribution (Ricard et al. 1989). An increase of the lithospheric thickness acts to reduce and to distribute the stress in a wider portion of the elastic layer, without modifying the pattern in a substantial manner. It should be noted that the new viscosity model (bottom) predicts a lower level of stress at present times, as a consequence of the faster relaxation in the upper mantle. These results do not depend on the size of the ice-sheet. 
The effects of a viscosity increase in the hard layer, with $C=50$, are shown in Fig. 18 for the old and new models (top and bottom respectively) and are compared with the findings for the uniform upper mantle $(C=1)$. The values of the lithospheric thickness are the same as those of Fig. 17, and both the lower and upper branch solutions are shown. For the lower branch, the viscosity increase in the hard layer $(C=50)$ causes a small increase, to a few tens of bars, in the maximum shear stress. The physics is similar to that already discussed for a viscosity increase in the lower mantle: the stiffening of the transition zone induces a delayed migration of stress in the lithosphere. This can have important implications on stress redistribution from upper mantle earthquakes, particularly in slabs. The amount of shear stress which migrates back in the lithosphere is reduced in comparison to what was obtained in Fig. 17 as a consequence of the smaller volume of the middle layer. On the other hand, the hard layer can be well resolved from the stress pattern even in the cases of mild viscosity contrasts, for both the Laurentide and Fennoscandia ice-sheets. The viscosity increase in the hard layer influences also the upper branch solutions for both the Laurentide and Fennoscandia. The stress pattern associated with the rebound in Fennoscandia is more sensitive than Laurentide to the viscosity increase in the hard layer, as shown by the third column $(c, f)$. This is due to the smaller size of the load which is more sensitive to the upper mantle structure in the transition zone.

For the Laurentide rebound and $d=120$ and $d=200 \mathrm{~km}$, Fig. 19 portrays the time evolution of the quantity $f(\theta, r)=\sigma_{\theta \theta}-\sigma_{r r}$ which describes the state of compression or tension with $f>0$ and $f<0$ respectively (Fleitout \& Froidevaux 1982). The pattern of $f(\theta, r)$ is extremely sensitive to the viscosity increase in the lower mantle. For the upper branch, we obtain a very high extension beneath the load (blue), in agreement with the recorded seismic pattern (Stein et al. 1979; Spada et al. 1991b). The outer portion of the lithosphere and the mantle is in compression (pink), except for a small region of the upper mantle that can be in extension if the lower mantle viscosity is sufficiently high. The extensional stresses are generally larger for the old model (intense blue, top). The size of the load and the thickness of the lithosphere produce minor influences in comparison to viscosity stratification in the lower mantle. We note that the hard layer can be responsible for a drastic modification in the stress pattern of the lithosphere, modifying the extensional configuration in the centre to a compressional one, (OM, LB, panels $a, b)$, owing to the coupled flexural properties of the interplaying lithosphere and stratified upper mantle. It must be noted that viscous relaxation in a lithosphere of $200 \mathrm{~km}$ can play an important role for forcings with periodicities of $10^{5}-10^{6}$ yr (Wolf 1986).

\section{CONCLUSIONS}

The effects of a viscosity increase in the transition zone have been studied for the whole set of signatures associated with post-glacial rebound. Starting with the lowest degree harmonic $l=2$, we have shown that the impact on $\dot{j}_{2}$ is minor as expected, because the hard layer is 'transparent' to the long-wavelength deformation associated with surface mass loading. The hard layer can be 'seen', on the contrary, by the high degree temporal variations of the geopotential coefficients, with $l>4$. Future satellite missions, such as LAGEOS II, will thus prove to be extremely significant for the inversion of the upper mantle rheological structure. The secular component of polar motion data is also extremely sensitive to the viscosity of the hard layer, owing to the dependence of the Liouville equations on coupled tidal and surface mass loading. A viscosity increase in the hard layer degrades our ability to fit polar wander data with a mild viscosity increase in the lower mantle. On the basis of this rotational datum, the hard layer favours an Earth model with a viscosity increase in the lower mantle. In order to balance such a global stiffening of the mantle, the new model with reduced viscosity in the upper mantle is preferred. This result is consistent with recent findings from geoid studies (Ricard et al. 1989), postglacial rebound (Lambeck et al. 1990) and theoretical studies on creep mechanisms in polycrystalline silicates (Ranalli 1991).

The hardening of the transition zone exerts significant effects on the vertical displacements and velocities, for both the two largest Pleistocenic ice-sheets. In particular the short-wavelength features of the rebound, such as the migration of the peripheral bulge, are strongly influenced by the flexural properties of the coupled system consisting in the lithosphere and upper mantle. A viscosity increase in the hard layer is responsible for a sharpening of the deformation pattern in the periphery, suggesting that geodetic surveying along continental margins in deglaciated areas is needed to provide an extra means for probing the upper mantle viscosity.

The horizontal component of the deformation is shown to be extremely sensitive to the transition zone, in terms of viscosity and chemical layering. As for the vertical displacements, the horizontal ones are sharpened by the stiffening below the $420 \mathrm{~km}$ discontinuity both in the near-field and in the far-field. Our results demonstrate that Very Long Baseline Interferometry (VLBI) data on intraplate deformation can play an important role in constraining the mechanical structure of the mantle below $420 \mathrm{~km}$.

The time evolution of the stress pattern in the outer elastic lithosphere is highly affected by the viscosity profile in the mantle. A stratified mantle is in fact responsible for a delayed focusing of the stress in the lithosphere. An isoviscous mantle is shown to behave quite differently, being essentially unable to maintain, after a few thousand of years after the end of melting, a sufficiently high level of stress to explain the recorded seismicity along the passive continental margins. The occurrence of earthquakes in these regions seems to require a stiff mantle that can be obtained by a viscosity increase in the transition zone or in the lower mantle. The seismic pattern in formerly glaciated areas, such as in Eastern Canada or Fennoscandia, may thus provide an extra geophysical observable to bound the viscosity profile in the mantle. Except for the $\dot{j}_{2}$ observations, we have demonstrated that the structure of the transition zone influences the whole set of geophysical observables due to post-glacial rebound, that should be jointly interpreted in order to constrain the viscosity profile.

Our results on the focusing of the stress in the lithosphere from surface mass redistribution suggest that the mechanical structure of transition zone may play a crucial role in other 
geodynamical processes, such as stress-diffusion from earthquake cycles and subduction (Bonafede, Dragoni \& Morelli 1986; Bonafede 1989). We have already seen that the presence of the hard layer tends in fact to induce a channel-like effect in the upper mantle, which could act as a stress-guide and control the recurrence times of seismic activity. Presently, models of earthquakes cycles do not account for the viscosity increase in the transition zone or lower mantle (Bonafede et al. 1986). Such a hardening of the mantle introduces an extra time-scale that could influence the characteristic recurrence times of the earthquakes. By the same token, the interaction between a subducting slab and the hard layer is expected to control the stress pattern and the related features such as topography, geoid anomalies and seismic pattern near Benioff zones. The results of present and future modelling efforts must be substantiated by further laboratory data which will provide the necessary physical constrained rheological parameters (Wang, Karato \& Fujino 1991).

\section{ACKNOWLEDGMENTS}

This work has been supported by the SCIENCE programme of the European Economical Community No. SC1*0456, by contract ASI 90-RS-89 and LAGEOS program of NASA. We are indebted to $D$. Wolf and $K$. Lambeck for their careful reviews and suggestions.

\section{REFERENCES}

Anderson, D. L., 1987. Thermally induced phase changes, lateral heterogeneity of the mantle, continental roots and deep-seated density anomalies, $J$. geophys. Res., 92, 13968-13980.

Anderson, D. L., 1989. Theory of the Earth, Blackwell Scientific Publ., Oxford.

Anderson, D. L. \& Bass, J. D., 1986. Transition region of the Earth's upper mantle, Nature, 320, 321-328.

Bonafede, M., 1989. The recurrence time of dislocation events on the antithetic margins of an elastic plate welded to a Maxwell viscoelastic half-space, J. geophys. Res., 94, 4159-4168.

Bonafede, M., Dragoni, M. \& Morelli, A., 1986. On the existence of a periodic dislocation cycle in horizontally layered viscoelastic models, J. geophys. Res., 81, 6396-6404.

Bungum, H., 1989. Earthquake occurrence and seismotectonics in Norway and surrounding areas, in Earthquakes at NorthAtlantic Passive Margins: Neotectonics and Postglacial Rebound, Series C: Mathematical and Physical Sciences, Vol. 266, pp. 501-519, eds Gregensen, S. \& Basham, P. W., Kluwer Academic Publishers, Dordrecht.

Calcagnile, G., 1982. The lithosphere-asthenosphere system in Fennoscandia, Tectonophysics, 90, 19-35.

Cheng, M. K., Eanes, R. J., Shum, C. K., Schutz, B. E. \& Tapley, B. D., 1989. Temporal variations in low degree zonal harmonics from Starlette orbit analysis, Geophys. Res. Lett., 16, 393-396.

Chinnery, M. A., 1975. The static deformation of the earth with a fluid core: a physical approach, Geophys. J. R. astr. Soc., 42, 461-474.

Colin, P. \& Fleitout, L., 1990. Topography of the ocean floor: thermal evolution of the lithosphere and interaction of deep mantle heterogeneities with the lithosphere, Geophys. Res. Lett., 17, 1961-1964.

Crossley, D. J. \& Gubbins, D., 1975. Static deformations of the earth's liquid core, Geophys. Res. Lett., 2, 1-4.
Dickman, S. R., 1981. Investigations of controversial polar motion features using homogeneous international latitude service data, J. geophys. Res., 86, 4094-4912.

Dost, B., 1990. Upper mantle structure under western Europe from fundamental and higher mode surface waves using the NARS array, Geophys. J. Int., 100, 131-151.

Duffy, H. \& Anderson, D. L., 1989. Seismic velocities in mantle minetals and the mineralogy of the upper mantle, J. geophys. Res., 94, 1895-1912.

Dziewonski, A. M. \& Anderson, D. L., 1981. Preliminary reference earth model, Phys. Earth planet. Inter., 25, 41-50.

Farrell, W. E., 1972. Deformation of the earth by surface loads, Rev. Geophys., 10, 761-797.

Fleitout, L. \& Froidevaux, C., 1982. Tectonics and topography for a lithosphere containing density heterogeneities, Tectonics, 1, $21-56$.

Fung, Y. C., 1965. Foundation of Solid Mechanics, Chap. 15, Prentice-Hall, Englewood Cliffs, NJ.

Grand, S. P., 1987. Tomographic inversion for shear velocity beneath the North American plate, J. geophys. Res., 92, $14065-14090$

Gubbins, D. \& Snieder, R., 1991. Dispersion of $P$ waves in subducted lithosphere: evidence for an ecologite layer, $J$. geophys. Res., 96.

Hasegawa, H. S. \& Hermann, R. B., 1989. A comparison of the source mechanism of the 1975 Laurentian Channel earthquake and the tsunamigenic 1929 Grand Banks event, in Earthquakes at North-Atlantic Passive Margins: Neotectonics and Postglacial Rebound, Series C: Mathematical and Physical Sciences, Vol. 266, pp. 547-562, eds Gregensen, S. \& Basham, P. W., Kluwer Academic Publishers, Dordrecht.

Hong, H. J., Yuen, D. A. \& Wu, J., 1990. Dynamical effects of a hard garnet layer in the transition zone on geophysical signatures: geoid anomalies and postglacial rebound, Phys. Earth planet. Inter., 64, 37-51.

James, T. S. \& Morgan, W. J., 1990. Horizontal motions due to post-glacial rebound, Geophys. Res. Lett., 17, 957-960.

Karato, S., 1989. Plasticity-crystal structure systematics in dense oxides and its implications for the creep strength of the Earth's deep interior: a preliminary result, Phys. Earth planet. Inter., 55, 234-240.

Karato, S., Paterson, M. S. \& Fitzgerald, J. D., 1986. Rheology of synthetic olivine aggregates: influence of grain size and water, J. geophys. Res., 91, 8151-8176.

Lambeck, K., 1980. The Earth's Variable Rotation, Chap. 5, Cambridge University Press, Cambridge, UK.

Lambeck, K., Johnston, P. \& Nakada, M., 1990. Holocene glacial rebound and sea-level change in NW Europe, Geophys. J. Int., $103,451-468$.

Lefftz, M. \& Legros, H., 1992. Some remarks about the rotations of a viscous planet and its homogeneous liquid core; linear theory, Geophys. J. Int., in press.

Longman, I. M., 1962. A Green's Function for determining the deformation of the earth under surface mass loads. 1. Theory, J. geophys. Res., 62, 845-850.

Longman, I. M., 1963. A Green's Function for determining the deformation of the earth under surface mass loads. 2. Computations and numerical results, J. geophys. Res., 68, 845-850.

Mackwell, S. J., Kohlstedt, D. L. \& Paterson, M. S., 1985. The role of water in the deformation of olivine single crystals, $J$. geophys. Res., 90, $11319-11333$.

Mackwell, S. J., Bai, Q. \& Kohlstedt, D. L., 1990. Rheology of olivine and the strength of the lithosphere, Geophys. Res. Lett., 17, 9-12.

Made, C. \& Jeanloz, R., 1990. The strength of mantle silicates at high pressure and room temperature: implications for the viscosity profile of the mantle, Nature, 348, 533-535. 
Mitrovica, J. X. \& Peltier, W. R., 1989. Pleistocene deglaciation and the global gravity field, $J$. geophys. Res., 94, $13651-13671$.

Montagner, J. P. \& Anderson, D. L., 1989. Constrained reference mantie model, Phys. Earth planet. Inter., 58, 205-227.

Morris, S., 1992. Rheometry using solid-state transformations occurring by nucleation and growth, Proc. R. Soc. Lond., A, in press.

Munk, W. H. \& MacDonald, G. J. F., 1960. The Rotation of the Earth, Cambridge University Press, New York.

Peltier, W. R., 1974. The impulse response of a Maxwell earth, Rev. Geophys., 12, 649-669.

Peltier, W. R., 1983. Constraint on deep mantle viscosity from Lageos acceleration data, Nature, 434-436.

Peltier, W. R., 1985. The LAGEOS constraint on deep mantle viscosity: results from a new normal mode method for the inversion of viscoelastic relaxation spectra, J. geophys. Res., 90, 9411-9421.

Ranalli, G., 1991. The microphysical approach to mantle rheology, in Glacial Isostasy, Sea Level and Mantle Rheology, Series C: Mathematical and Physical Sciences, vol. 334, eds Sabadini, R., Lambeck, K. \& Boschi, E., Kluwer Academic Publishers, Dordrecht.

Ricard, Y. \& Sabadini, R., 1990. Rotational instabilities of the Earth induced by mantle density anomalies, Geophys. Res. Lett., 17, 627-630.

Ricard, Y., Vigny, C. \& Froidevaux, C., 1989. Mantle heterogeneities, geoid, and plate motion: a Monte Carlo inversion, J. geophys. Res., 94, 13739-13754.

Sabadini, R. \& Peltier, W. R., 1981. Pleistocene deglaciation and the Earth's rotation: implications for mantle viscosity, Geophys. J. R. astr. Soc., 66, 553-578.

Sabadini, R. \& Yuen, D. A., 1989. Mantle stratification and long-term polar wander, Nature, 339, 373-375.

Sabadini, R., Yuen, D. A. \& Boschi, E., 1982. Polar wander and the forced responses of a rotating, multilayered, viscoelastic planet, J. geophys. Res., 87, 2885-2903.

Sabadini, R., Yuen, D. A. \& Boschi, E., 1984a. The effects of post-seismic motions on the moment of inertia of a stratified viscoelastic earth with an asthenosphere, Geophys. J. R. astr. Soc., 79, 727-746.

Sabadini, R., Yuen, D. A. \& Boschi, E., 1984b. A comparison of the complete and truncated versions of the polar wander equations, J. geophys. Res., 89, 7609-7620.

Sabadini, R., Yuen, D. A. \& Gasperini, P., 1988. Mantle rheology and satellite signatures from present-day glacial forcing, $J$. geophys. Res., 93, 437-447.

Spada, G., Sabadini, R. \& Yuen, D. A., 1991a. The dynamical influences of a hard transition zone on post-glacial uplift and rotational signatures, Glacial Isostasy, Sea-level and Mantle Rheology, Series C: Mathematical and Physical Sciences, Vol.
334, pp. 121-141, eds Sabadini, R., Lambeck, K. \& Boschi, E., Kluwer Academic Publishers, Dordrecht.

Spada, G., Yuen, D. A., Sabadini, R. \& Boschi, E., 1991b. Lower-mantle viscosity constrained by seismicity around deglaciated regions, Nature, 351, 53-55.

Spada, G., Yuen, D. A., Sabadini, R., Morin, P. J. \& Gasperini, P., 1990. A computer-aided, algebraic approach to the post-glacial rebound problem, Mathematica J., 1, 65-69.

Stein, S., Sleep, N., Geller, R., Wang, S. \& Kroger, G., 1979. Earthquakes along the passive margins of eastern Canada, Geophys. Res. Lett., 5, 537-540.

Takeuchi, H., Saito, M. \& Kobayashi, N., 1962. Statical deformations and the free oscillations of a model earth, $J$. geophys. Res., 67, 1141-1154.

Walcott, R. J., 1972. Late Quaternary vertical movements in Eastern North America: quantitative evidence of glacioisostatic rebound, Rev. Geophys., 10, 849-884.

Wang, Z., Karato, S. \& Fujino, K., 1991. High-temperature plasticity of garnet, II, EOS, Trans. Am. geophys. Un., 72, 452.

Widmer, R., Masters, G. \& Gilbert, F., 1991. Spherically symmetric attenuation within the Earth from normal mode data, Geophys. J. Int., 104, 541-553.

Wolf, D., 1986. Glacio-isostatic adjustment in Fennoscandia revisited, J. Geophys., 59, 42-48.

Wolf, D., 1987. An upper bound on lithosphere thickness from glacio-isostatic adjustment in Fennoscandia, J. Geophys., 61, $141-149$.

Wolfram, S. et al., 1988. Mathematica, a System for doing Mathematics by Computer, Addison-Wesley, Redwood, CA.

Wu, P., 1978. The response of a Maxwell earth to applied surface loads: Glacial isostatic adjustment, MSc thesis, University of Toronto.

Wu, P. \& Peltier, W. R., 1982. Glacial isostatic adjustment and the free air gravity anomaly as a constraint of deep mantle viscosity, Geophys. J. R. astr. Soc., 74, 377-449.

Wu, J. \& Yuen, D. A., 1991. Post-glacial relaxation of a viscously stratified compressible mantle, Geophys. J. Int., 104, 331-349.

Yoder, C. F., Williams, J. G., Dickey, J. O., Schutz, B. E., Eanes, R. J. \& Tapley, B. D., 1983. Secular variation of the Earth's gravitational harmonic $J_{2}$ coefficient from Lageos and non-tidal acceleration of Earth rotation, Nature, 303, 757-762.

Yuen, D. A. \& Peltier, W. R., 1980. Mantle plumes and the thermal stability of the D" layer, Geophys. Res. Lett., 7, 625-628.

Yuen, D. A., Sabadini, R. \& Boschi, E., 1982. Viscosity of the lower mantle as inferred from rotational data, J. geophys. Res., 87, $10745-10762$.

Yuen, D. A., Sabadini, R., Gasperini, P. \& Boschi, E., 1986. On transient rheology and glacial isostasy, J. geophys. Res., 91, 11 420-11438.

\section{APPENDIX}

We present here the analytical form of both the fundamental matrix $Y(r, s)$ and its inverse $Y^{-1}(r, s)$, as a function of the physical parameters which characterize each of layers. The parameters have the following meaning: $l=$ harmonic order of Legendre polynomial $P_{f}(\cos \theta), r=$ distance from the centre of the Earth, $\mu(s)=\mu s /(s+\mu / v)$ complex shear modulus of the layer, $s=$ complex Laplace variable, $v=$ dynamic viscosity, $\rho=$ density of the layer, $g(r)=$ gravity field, and $G=$ universal gravitational constant. 
The direct matrix, containing a set of six linearly independent solution vectors, is given by

$$
Y(r, s)=\left(\begin{array}{cccccc}
\frac{l r^{l+1}}{2(2 l+3)} & r^{l-1} & 0 & \frac{(l+1) r^{-l}}{2(2 l-1)} & r^{-l-2} & 0 \\
\frac{(l+3) r^{l+1}}{2(2 l+3)(l+1)} & \frac{r^{l-1}}{l} & 0 & \frac{(2-l) r^{-l}}{2 l(2 l-1)} & \frac{-r^{-l-2}}{(l+1)} & 0 \\
\frac{l \rho g(r) r^{l+1}}{2(2 l+3)}+ & \frac{\rho g(r)}{r^{-l+1}} & -\rho r^{l} & \frac{(l+1) \rho g(r)}{2(2 l-1) r^{l}+} & \frac{\rho g(r)}{r^{l+2}+} & \frac{-\rho}{r^{l+1}} \\
\frac{\left(l^{2}-l-3\right) \mu(s)}{(2 l+3) r^{l}} & +\frac{2(l-1) \mu(s)}{r^{-l+2}} & \frac{\left(-l^{2}-3 l+1\right) \mu(s)}{(2 l-1) r^{l+1}}-\frac{2(l+2) \mu(s)}{r^{l+3}} & \\
\frac{l(l+2) \mu(s) r^{l}}{(2 l+3)(l+1)} & \frac{2(l-1) \mu(s) r^{l-2}}{l} & 0 & \frac{\left(l^{2}-1\right) \mu(s)}{l(2 l-1) r^{l+1}} & \frac{2(l+2) \mu(s)}{(l+3) r^{l+3}} & 0 \\
0 & 0 & -r^{l} & 0 & 0 & \frac{-1}{r^{l+1}} \\
\frac{2 \pi G \rho l r^{l+1}}{2 l+3} & 4 \pi G \rho r^{l-1} & \frac{-(2 l+1)}{r^{-l+1}} & \frac{2 \pi G \rho(l+1)}{(2 l-1) r^{l}} & \frac{4 \pi G \rho}{r^{l+2}} & 0
\end{array}\right) .
$$

The inversion of $Y(r, s)$ has been performed analytically by means of the algebraic manipulator Mathematica (Wolfram et al. 1988) on a NeXT computer (Spada et al. 1990). It is useful to define

$$
\bar{Y}=\left(\begin{array}{cccccc}
\frac{\rho g(r) r}{\mu(s)}-2(l+2) & 2 l(l+2) & -\frac{r}{\mu(s)} & \frac{l r}{\mu(s)} & \frac{\rho r}{\mu(s)} & 0 \\
-\frac{\rho g(r) r}{\mu(s)}+\frac{2\left(l^{2}+3 l+1\right)}{l+1} & 2\left(l^{2}-1\right) & \frac{r}{\mu(s)} & \frac{(2-l) r}{\mu(s)} & -\frac{\rho r}{\mu(s)} & 0 \\
4 \pi G \rho & 0 & 0 & 0 & 0 & 1 \\
\frac{\rho g(r) r}{\mu(s)}+2(l-1) & 2\left(l^{2}-1\right) & -\frac{r}{\mu(s)} & -\frac{(l+1) r}{\mu(s)} & \frac{\rho r}{\mu(s)} & 0 \\
-\frac{\rho g(r) r}{\mu(s)}-\frac{2\left(l^{2}-l-3\right)}{l} & -2 l(l+2) & \frac{r}{\mu(s)} & \frac{(l+3) r}{\mu(s)} & -\frac{\rho r}{\mu(s)} & 0 \\
4 \pi G \rho r & 0 & 0 & 0 & 2 l+1 & -r
\end{array}\right)
$$

and to introduce a diagonal matrix $D$ whose non-zero elements are $(i=1, \ldots, 6)$

$D_{i i}=\left[\frac{(l+1) r^{-l-1}}{(2 l+1)}, \frac{l(l+1) r^{-l+1}}{2(2 l+1)(2 l-1)}, \frac{r^{-l+1}}{(2 l+1)}, \frac{l r^{l}}{(2 l+1)}, \frac{l(l+1) r^{l+2}}{2(2 l+1)(3 l+2)}, \frac{-r^{l+1}}{(2 l+1)}\right]$.

The inverse and the determinant of $Y(r, s)$ are respectively given by

$Y^{-1}(r, s)=D \bar{Y}$

and

$|Y(r, s)|=-\left[\frac{l(l+1)}{(2 l+1)^{3 / 2}} \frac{r^{3}}{\mu(s)}\right]^{-2}$

The decomposition in 'elastic' and 'viscous' parts given in the text (equations 5a and 5b), are simply related to the structure of both the direct and the inverse fundamental matrices, which can be expressed in the form of first-order matrix polynomials in $\mu(s)$ and $\mu(s)^{-1}$ respectively. 